\title{
Claudin 11 regulates bone homeostasis via bidirectional EphB4-EphrinB2 signaling
}

\author{
Jong Min Baek', Yoon-Hee Cheon', Sung Chul Kwak', Hong Young Jun², Kwon-Ha Yoon ${ }^{2,3}$, Myeung Su Lee ${ }^{2,4}$ and \\ Ju-Young Kim $\mathbb{1}^{2}$
}

\begin{abstract}
Claudins (Cldns) are well-established components of tight junctions (TJs) that play a pivotal role in the modulation of paracellular permeability. Several studies have explored the physiologic aspects of Cldn family members in bone metabolism. However, the effect of Cldn11, a major component of central nervous system myelin, on bone homeostasis has not been reported. In this study, we demonstrate that Cldn11 is a potential target for bone disease therapeutics as a dual modulator of osteogenesis enhancement and osteoclastogenesis inhibition. We found that Cldn11 played a negative role in the receptor activator of nuclear factor kappa B ligand-induced osteoclast (OC) differentiation and function by downregulating the phosphorylated form of extracellular signal-regulated kinase (ERK), Bruton's tyrosine kinase, and phospholipase C gamma 2, in turn impeding c-Fos and nuclear factor in activated T cell c1 expression. The enhancement of osteoblast (OB) differentiation by positive feedback of Cldn11 was achieved through the phosphorylation of Smad1/5/8, ERK, and c-Jun amino-terminal kinase. Importantly, this Cldn11-dependent dual event in bone metabolism arose from targeting EphrinB2 ligand reverse signaling in OC and EphB4 receptor forward signaling in OB. In agreement with these in vitro effects, subcutaneous injection of Cldn11 recombinant protein exerted anti-resorbing effects in a lipopolysaccharide-induced calvarial bone loss mouse model and increased osteogenic activity in a calvarial bone formation model. These findings suggest that Cldn11 is a novel regulator in bone homeostasis.
\end{abstract}

\section{Introduction}

A large family of integral transmembrane proteinsclaudin (Cldn), occludin, tricellulin, and junction adhesion molecules-has been identified as the components of tight junctions (TJs) physiologically involved in the establishment of multicellular organisms and maintenance of biological compartment systems ${ }^{1,2}$. Beyond their well-characterized roles as junctional complexes, TJs have emerged as critical regulators of cellular functions through the regulation of transcellular transit of ions,

Correspondence: Myeung Su Lee (ckhlms@wku.ac.kr) or Ju-Young Kim (kimjy1014@gmail.com)

'Department of Anatomy, School of Medicine, Wonkwang University, Iksan, Republic of Korea

${ }^{2}$ Medical Convergence Research Center, Wonkwang University Hospital, Iksan, Republic of Korea

Full list of author information is available at the end of the article small molecules, and water and control of cell polarity, proliferation, differentiation, and tumor suppression ${ }^{1-4}$.

Among the membrane proteins of TJs, Cldns are key molecular components responsible for core structure formation in TJ strands by functioning as barriers or pores to modulate paracellular permeability ${ }^{5}$. Through previous studies employing loss of function and gain of function in mice, it was revealed that Cldn11-knockout mice showed abnormal phenotypes in terms of the structure of TJ strands in central nervous system myelin, Sertoli cells, and strial marginal cells of the inner ear ${ }^{6-8}$. That said, there is substantial evidence that Cldn exerts non-canonical effects on intracellular communication, thereby mediating cell proliferation and differentiation. Recently, Shimobaba et al. ${ }^{9}$ proved that Cldn18 plays a negative role in the proliferation of lung adenocarcinoma A549 cells via the phosphatidylinositol-3 kinase/phosphoinositide- 
dependent protein kinase-1/Akt pathway ${ }^{9}$. For positive regulation, Cldn1 leads to induction of the epithelial-mesenchymal transition in human liver cells by triggering activation of the c-Abl-Ras-extracellular signalregulated kinase (ERK) signaling axis, serving as a candidate biomarker associated with liver cancer metastasis ${ }^{10}$.

Various studies have also revealed a clear correlation between Cldn and bone-resorbing osteoclasts (OCs) or bone-forming osteoblasts (OBs). Cldn18 deficiency significantly promotes $\mathrm{OC}$ differentiation in response to the receptor activator of nuclear factor kappa B ligand (RANKL) to drive the differentiation of OC precursors, such as the differentiation of bone marrow macrophages (BMMs) into tartrate-resistant acid phosphatase (TRAP)positive multinucleated cells (MNCs) in vitro ${ }^{11}$. In agreement with this observation, Cldn18-knockout mice suffer from an impaired skeletal state with markedly low bone mass derived from immoderate OC activity ${ }^{11-13}$. Additionally, in the absence of Cldn1 in mouse osteoblastic cell lines (MC3T3-E1) induced by lentiviral short hairpin RNA, the enzymatic activity of alkaline phosphatase (ALP) and expression of master transcription factors associated with bone formation, including Runx 2 and osterix, are negatively regulated ${ }^{14}$. Therefore, we believe that investigating the role of other subtypes of Cldn in bone metabolism is important to guide further clinical trials and provide novel clues on the pathogenesis of bone-related disorders, such as osteoporosis.

To propose a potential correlation of one of the subtypes of the Cldn family with bone metabolism, we performed various in vitro and in vivo studies using gain of function and loss of function of Cldn11, which belongs to the Cldn group. The present study was designed to provide evidence for the effect of Cldn 11 on the differentiation and function of OCs and OBs, suggesting that Cldn11 might be a crucial modulator in the maintenance of bone homeostasis.

\section{Materials and methods \\ Reagents and antibodies}

Recombinant soluble human macrophage colony stimulating factor (M-CSF), RANKL, and interleukin-1 (IL-1) were obtained from PeproTech EC Ltd. (London, UK), and recombinant mouse Fc-EphrinB2 and EphB4 were purchased from $R \& D$ Systems (Minneapolis, MN, USA). Cldn11 recombinant protein used for in vivo study was purchased from MyBioSource (San Diego, CA, USA). Vitamin $\mathrm{D}_{3}\left(\mathrm{Vit}_{3}\right)$, prostaglandin $\mathrm{E}_{2}\left(\mathrm{PGE}_{2}\right)$, ascorbic acid (AA), $\beta$-glycerol phosphate ( $\beta$-gly), and lipopolysaccharide (LPS) were purchased from Sigma-Aldrich (St. Louis, MO, USA). A monoclonal $\beta$-actin (A5441) and phospho-Bruton's tyrosine kinase (Btk; GTX61791) antibodies were obtained from Sigma-Aldrich (St. Louis, MO,
USA) and GeneTex (Irvine, CA, USA), respectively. Antibodies against phospho-p38 (\#9211), p38 (\#9212), phospho-ERK (\#9101), ERK (\#9102), phospho-c-Jun amino-terminal kinase (JNK; \#9251), JNK (\#9252), phospho-IкB (\#2859), phospho-Akt (\#9271), Akt (\#9272), Btk (\#3533), and phospho-Smad1/5/8 (\#9511) were purchased from Cell Signaling Technology Inc. (Beverly, MA, USA). Antibodies against c-Fos (sc-7202), nuclear factor of activated T cells c1 (NFATc1; sc-7294), ІкB (sc-371), phospho-phospholipase C gamma 2 (PLC 2; sc-101785), PLCY2 (sc-5283), Smad1/5/8 (sc-6031), Cldn11 (sc271232), EphrinB2 (sc-15397) and EphB4 (sc-5536), and antibody-coated protein A/G-Agarose (sc-2003) were purchased from Santa Cruz Biotechnology (Santa Cruz, CA, USA). Alexa Fluor 488 goat anti-mouse IgG (R37120), Alexa Fluor 568 goat anti-rabbit (A-11011), and 4',6-diamidino-2-phenylindole (DAPI) were purchased from Life Technologies Corporation (Carlsbad, CA, USA). Fetal bovine serum (FBS), $\alpha$-minimum essential medium $(\alpha-M E M)$, penicillin, and streptomycin were purchased from Gibco BRL (Grand Island, NY, USA). All other chemicals were of analytical grade or complied with the standards required for cell culture experiments.

\section{Experimental animals}

Five-week-old male Institute of Cancer Research (ICR) mice were purchased from Samtako Co. Ltd. (Osan, Korea) and kept at $22-24{ }^{\circ} \mathrm{C}$ and $55-60 \%$ humidity with a $12 \mathrm{~h}$ light/dark cycle. All experiments were conducted according to the guidelines of the Institutional Animal Care and Use Committee of Wonkwang University (Approved number: WKU16-7).

\section{In vitro $O C$ culture and TRAP assay}

Bone marrow cells (BMCs) were obtained from 5-weekold male ICR mice by flushing the femurs and tibias with $\alpha$-MEM supplemented with $10 \%$ FBS, penicillin (100 U/ $\mathrm{mL})$, and streptomycin $(100 \mu \mathrm{g} / \mathrm{mL})^{15}$. To obtain BMMs, BMCs were seeded on culture dishes in $\alpha$-MEM supplemented with $10 \%$ FBS and M-CSF $(10 \mathrm{ng} / \mathrm{mL})$ and cultured for 1 day. Non-adherent cells were transferred to $10-\mathrm{cm}$ petri dishes and further cultured in the presence of M-CSF $(30 \mathrm{ng} / \mathrm{mL})$ for 3 days. After the non-adherent cells were removed, the adherent cells were used as BMMs, which are OC precursors. To generate OCs from these BMMs, the cells were seeded in a 48-well plate $\left(3.5 \times 10^{4}\right.$ cells/well $)$ in complete medium containing M-CSF $(30 \mathrm{ng} / \mathrm{mL})$ and RANKL $(100 \mathrm{ng} / \mathrm{mL})$ and cultured for 4 days. The cells were fixed in $3.7 \%$ formalin for $10 \mathrm{~min}$, permeabilized with $0.1 \%$ Triton X-100, and then stained with TRAP (Sigma). TRAP-positive MNCs with more than five nuclei were counted as OCs. 


\section{Survival and resorption pit assay}

Mature OCs were prepared from the co-culture of BMCs and primary OBs as described previously ${ }^{16}$. Briefly, BMCs $\left(1 \times 10^{7}\right.$ cells $)$ and primary OBs $\left(1 \times 10^{6}\right.$ cells $)$ were cultured in collagen gel-coated culture dishes in the presence of $10^{-8} \mathrm{M} \mathrm{VitD}{ }_{3}$ and $10^{-6} \mathrm{M} \mathrm{PGE}_{2}$ for 9 days. Co-cultured mature OCs were detached using $0.1 \%$ collagenase at $37^{\circ} \mathrm{C}$ for $10 \mathrm{~min}$ and re-seeded in 48-well plates, hydroxyapatite-coated plates (Corning, NY, USA), and dentin slices. After $1 \mathrm{~h}$, the cells were transfected with the indicated retrovirus or small interfering RNA (siRNA) as described above and further cultured in the presence of RANKL ( $100 \mathrm{ng} / \mathrm{mL}$ ). After $48 \mathrm{~h}$, the cells re-seeded in 48well plates were stained with TRAP solution to detect the survival of OCs. The cells re-seeded in hydroxyapatitecoated plates and dentin slices were completely removed using $10 \%$ sodium hypochlorite after 24 and $48 \mathrm{~h}$, respectively. Additionally, dentins were stained with hematoxylin to detect resorption pits. The total number of resorption pits was determined under a microscope and were quantified using Image-Pro Plus version 4.0 (Media Cybernetics, Silver Spring, MD, USA).

\section{In vitro $\mathrm{OB}$ culture and assays}

Primary OBs were isolated from the calvaria of neonatal mice and cultured as previously described ${ }^{16}$. To culture $\mathrm{OBs}$, the calvaria of neonatal mice were digested with $0.1 \%$ collagenase and $0.2 \%$ dispase five times, and cells isolated in the last three digestions were combined and cultured in $\alpha$-MEM containing 10\% FBS, $100 \mathrm{U} / \mathrm{mL}$ penicillin, and $100 \mu \mathrm{g} / \mathrm{mL}$ streptomycin. To measure ALP activity, primary OBs were inoculated at a density of $2 \times$ $10^{4}$ cells/well and cultured in the absence or presence of $50 \mu \mathrm{g} / \mathrm{mL}$ AA and $10 \mathrm{mM} \beta$-gly. On differentiation day 7 , the cells were sonicated in $50 \mathrm{mM}$ Tris- $\mathrm{HCl}$ buffer $(\mathrm{pH}$ 7.4) containing $1 \%$ Triton $\mathrm{X}-100,150 \mathrm{mM} \mathrm{NaCl}$, and 1 $\mathrm{mM}$ EDTA. Then, $100 \mu \mathrm{L}$ of substrate ( $p$-nitrophenylphosphate) was added to the cells, and the plate was incubated for $30 \mathrm{~min}$ at $37^{\circ} \mathrm{C}$. The amount of $p$-nitrophenol released was determined by measuring the absorbance at $405 \mathrm{~nm}$ using a microplate reader. For ALP staining, cells were fixed in $70 \%$ ethanol and stained for 10-20 min with a solution containing $0.01 \%$ naphthol, AS-MX phosphate, $1 \% N, N$-dimethyl formamide, and $0.06 \%$ fast blue BB. For alizarin red S (ARS) staining, on differentiation day 21 , cultured cells were fixed in $3.7 \%$ formalin and stained for $10 \mathrm{~min}$ with $2 \%$ ARS (pH 4.2). Bound ARS was dissolved in a $10 \%$ cetylpyridinium chloride monohydrate solution (CPC; $\mathrm{pH} 7.0$ ). Absorbance was measured at $545 \mathrm{~nm}$ using a microplate reader.

\section{Retroviral gene transfection}

Packaging of the retroviral vectors pMX-IRES-EGFP and pMX-Cldn11-IRES-EGFP was performed using transient transfection of these pMX vectors into Platinum-E retroviral packaging cells using $\mathrm{X}$ tremeGENE 9 (Roche, Nutley, NJ, USA) according to the manufacturer's protocol. After incubation in fresh medium for 2 days, the culture supernatants of the retrovirus-producing cells were collected. For retroviral infection, non-adherent BMCs were cultured in M-CSF $(30 \mathrm{ng} / \mathrm{mL})$ for 2 days. The BMMs were incubated with viral supernatant pMX-IRES-EGFP and pMX-Cldn11IRES-EGFP virus-producing plat-E cells together with polybrene $(10 \mu \mathrm{g} / \mathrm{mL})$ and $\mathrm{M}-\mathrm{CSF}(30 \mathrm{ng} / \mathrm{mL})$ for $6 \mathrm{~h}$. The infection efficiency of the retrovirus was determined by green fluorescent protein expression and was always $>80 \%$. After infection, the BMMs were induced to differentiate in the presence of M-CSF $(30 \mathrm{ng} / \mathrm{mL})$ and RANKL (50 or $100 \mathrm{ng} / \mathrm{mL}$ ) for 4 days. The forced expression of each construct (green fluorescent protein) and $\mathrm{OC}$ formation was detected using a fluorescence microscope and TRAP staining, respectively.

\section{siRNA gene transfection}

Mouse Cldn11 and negative control siRNA were designed and synthesized by Ambion (Austin, TX, USA). Mouse BMMs or OBs were transfected with $10 \mathrm{nM}$ of siRNA oligonucleotides using Lipofectamine 3000 (Invitrogen, San Diego, CA, USA) according to the manufacturer's instructions. Briefly, after incubating BMMs or OBs in $\alpha$-MEM containing $10 \%$ FBS, siRNA $(10 \mathrm{nM})$ Lipofectamine $3000(0.5 \mu \mathrm{L} / 48$-well or $3 \mu \mathrm{L} / 6$-well $)$ mixtures in Opti-MEM (Invitrogen) were added to BMMs or OBs and incubated for 6 or $8 \mathrm{~h}$, respectively. The medium was then replaced with fresh complete $\alpha$-MEM, and the cells were further cultured with an appropriate osteoclastogenic or osteogenic medium.

\section{Quantitative real-time RT-PCR}

Total RNA was isolated with Trizol reagent (Invitrogen) according to the manufacturer's instructions. To obtain cDNA, $2 \mu \mathrm{g}$ of equal amounts of total RNA were reverse transcribed into cDNA using SuperScript II Reverse Transcriptase (Invitrogen). Real-time reverse transcription-PCR (RT-PCR) was performed in a $20-\mu \mathrm{L}$ reaction mixture containing $10 \mu \mathrm{L}$ of SYBR Green Premix (Bioneer Co., Daejeon, Korea), 10 pmol of forward primer, $10 \mathrm{pmol}$ of reverse primer, and $1 \mu \mathrm{g}$ of cDNA using a Exicycler $^{\text {rm }} 96$ Real-Time Quantitative Thermal Block (Bioneer Co.). The primer sets used in quantitative realtime RT-PCR are shown in Table 1. The mouse GAPDH gene was used as the internal control. The amplification parameters consisted of an initial denaturation step at $95^{\circ}$ $\mathrm{C}$ for $5 \mathrm{~min}$, and then 40 cycles of denaturation at $95^{\circ} \mathrm{C}$ for $1 \mathrm{~min}$, annealing at $60^{\circ} \mathrm{C}$ for $30 \mathrm{sec}$, and extension at $72^{\circ} \mathrm{C}$ for $1 \mathrm{~min}$. The specificity of the SYBR green assays was confirmed by melting-point analysis. Expression data 
Table 1 Primer sequences used for quantitative real-time RT-PCR analysis

\begin{tabular}{|c|c|c|}
\hline Gene & Gene & Primer sequence $\left(5^{\prime} \rightarrow 3^{\prime}\right)$ \\
\hline \multirow[t]{2}{*}{ GAPDH } & Forward & TCAAGAAGGTGGTGAAGCAG \\
\hline & Reverse & AGTGGGAGTTGCTGTTGAAGT \\
\hline \multirow[t]{2}{*}{ DC-STAMP } & Forward & TCCTCCATGAACAAACAGTTCCA \\
\hline & Reverse & AGACGTGGTTTAGGAATGCAGCTC \\
\hline \multirow[t]{2}{*}{ OC-STAMP } & Forward & ATGAGGACCATCAGGGCAGCCACG \\
\hline & Reverse & GGAGAAGCTGGGTCAGTAGTTCGT \\
\hline \multirow[t]{2}{*}{ Cathepsin K } & Forward & CCAGTGGGAGCTATGGAAGA \\
\hline & Reverse & CTCCAGGTTATGGGCAGAGA \\
\hline \multirow[t]{2}{*}{ OSCAR } & Forward & GGAATGGTCCTCATCTCCTT \\
\hline & Reverse & TCCAGGCAGTCTCTTCAGTTT \\
\hline \multirow[t]{2}{*}{ Atp6rOd2 } & Forward & GACCCTGTGGCACTITITGT \\
\hline & Reverse & GTGTTTGAGCTTGGGGAGAA \\
\hline \multirow[t]{2}{*}{ c-Fos } & Forward & GGTGAAGACCGTGTCAGGAG \\
\hline & Reverse & TATTCCGTTCCCTTCGGATT \\
\hline \multirow[t]{2}{*}{ NFATC1 } & Forward & GAGTACACCTTCCAGCACCTT \\
\hline & Reverse & TATGATGTCGGGGAAAGAGA \\
\hline \multirow[t]{2}{*}{ TRAP } & Forward & TCATGGGTGGTGCTGCT \\
\hline & Reverse & GCCCACAGCCACAAATCT \\
\hline \multirow[t]{2}{*}{ B3-integrin } & Forward & GGAGTGGCTGATCCAGATGT \\
\hline & Reverse & TCTGACCATCTTCCCTGTCC \\
\hline \multirow[t]{2}{*}{ av-integrin } & Forward & ACAAGCTCACTCCCATCACC \\
\hline & Reverse & ATATGAGCCTGCCGACTGAC \\
\hline \multirow[t]{2}{*}{ Runx2 } & Forward & TGCCTTCAGCACCCTATACC \\
\hline & Reverse & AGGTTGGAGGCACACATAGG \\
\hline \multirow[t]{2}{*}{$A L P$} & Forward & GCTGATCATTCCCACGTTT \\
\hline & Reverse & ACCATATAGGATGGCCGTGA \\
\hline \multirow[t]{2}{*}{ OPN } & Forward & TCTGATGAGACCGTCACTGC \\
\hline & Reverse & CCTCAGTCCATAAGCCAAGC \\
\hline \multirow[t]{2}{*}{ OCN } & Forward & CTTGGGTTCTGACTGGGTGT \\
\hline & Reverse & ACCTTATTGCCCTCCTGCTT \\
\hline \multirow[t]{2}{*}{ Colla } & Forward & TGTGTTCCCTACTCAGCCGT \\
\hline & Reverse & CATCGGTCATGCTGTCTCCAA \\
\hline \multirow[t]{2}{*}{$B S P$} & Forward & AGGGAACTGACCAGTGTTGG \\
\hline & Reverse & ACTCAACGGTGCTGCTITIT \\
\hline \multirow[t]{2}{*}{$O P G$} & Forward & ACCTCACCACAGAGCAGCTT \\
\hline & Reverse & GCTCGATTTGCAGGTCTTTC \\
\hline \multirow[t]{2}{*}{ RANKL } & Forward & GACTCCATGAAAACGCAGGT \\
\hline & Reverse & TGTGTTGCAGTTCCTTCTGC \\
\hline
\end{tabular}

Table 1 continued

\begin{tabular}{lll}
\hline Gene & Gene & Primer sequence $\left(\mathbf{5}^{\prime} \rightarrow \mathbf{3}^{\prime}\right)$ \\
\hline Cldn11 & Forward & TGACCTGCAGCTACACCATC \\
& Reverse & GGG GTT TGC AGT GGT AGA GA \\
EphrinB2 & Forward & TCTGTGTGGAAGTACTGTTGGGGATT \\
& Reverse & TGTACCAGCTTCTAGCTCTGGACGTCTT \\
EphB4 & Forward & CGTCCTGATGTCACCTATACCTTGAGG \\
& Reverse & GAGTACTCAACTTCCCTCCCATTGCTCT \\
\hline
\end{tabular}

$R T-P C R$ reverse transcription-PCR

were calculated from the cycle threshold $\left(C_{\mathrm{t}}\right)$ value using the $C_{\mathrm{t}}$ method.

\section{Co-immunoprecipitation and western blot analyses}

Total cellular protein was lysed using extraction buffer containing $50 \mathrm{mM}$ Tris- $\mathrm{HCl}, 150 \mathrm{mM} \mathrm{NaCl}, 5 \mathrm{mM}$ EDTA, $1 \%$ Triton X-100, $1 \mathrm{mM}$ sodium fluoride, $1 \mathrm{mM}$ sodium vanadate, $1 \%$ deoxycholate, and protease inhibitors ${ }^{17}$. Protein content was measured using a Bio-Rad colorimetric protein assay kit (Bio-Rad Laboratories Inc., Hercules, CA, USA). Equal amounts of protein were immunoprecipitated with the indicated antibodies, and whole-cell lysates were run on $12 \%$ sodium dodecyl sulfate-polyacrylamide gel electrophoresis gels and were transferred by electroblotting onto polyvinylidene difluoride membranes (Millipore, Bedford, MA, USA). The membranes were blocked with $5 \%$ nonfat milk in Tris-buffered saline contacting $0.1 \%$ Tween-20 (TBST) for $1 \mathrm{~h}$ before blotting with the primary antibodies (EphrinB2 and phosphor-Btk, 1:500; EphB4, 1:250; the other antibodies, 1:1000 dilution) for $2 \mathrm{~h}$ at room temperature. The membranes were washed in TBST and incubated for $1 \mathrm{~h}$ with horseradish peroxidase-conjugated sheep anti-mouse or donkey anti-rabbit immunoglobulin antibodies (1:3000 dilution). Specific signals were detected using the Western Chemiluminescent HRP substrate kit

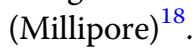

\section{Immunofluorescence staining}

OCs and OBs grown on glass slides in a 12-well plate were fixed with $4 \%$ paraformaldehyde, permeabilized with $0.1 \%$ Triton X-100, and incubated in $2.5 \%$ BSA solution for the blocking stage. The cells were stained with primary antibodies against Cldn11, EphrinB2, and EphB4 (1:1000) at room temperature for $1 \mathrm{~h}$ and $4{ }^{\circ} \mathrm{C}$ overnight, incubated with Alexa Fluor 488 goat anti-mouse IgG and Alexa Fluor 568 goat anti-rabbit (1:200) at room temperature for $2 \mathrm{~h}$, and then stained with DAPI solution for $1 \mathrm{~min}$. The stained cells were observed under a laser scanning 
confocal microscope (Olympus FV1200, Tokyo, Japan) to detect the co-localization of Cldn11 and EphrinB2/EphB4. Images were analyzed by Image-Pro Plus version 4.0.

\section{Construction and analysis of LPS-mediated calvarial bone erosion model}

To determine the effect of Cldn11 in vivo, 5-week-old male ICR mice were divided into three groups of six mice and injected in the calvaria subcutaneously with LPS (500 $\mu \mathrm{g} /$ mouse) or an equal volume of phosphate-buffered saline (PBS) on days 2 and 6 . Recombinant protein of Cldn11 (0.6 mg/mouse) was injected subcutaneously daily for 9 days into the calvaria. The mice were anesthetized 9 days after the treatment, and the calvarial bones were washed with PBS and fixed with $4 \%$ paraformaldehyde for 1 day. The calvaria were analyzed using high-resolution micro-CT (NFR-Polaris-S160; Nanofocus Ray, Iksan, Korea) to obtain 3D images (INFINITT-Xelis software; INFINITT Healthcare, Seoul, Korea). The images were acquired at $65 \mathrm{kVp}, 90 \mu \mathrm{A}$, and $313 \mathrm{~ms} /$ frame, and there were 512 views. The reconstruction image size was $1024 \times 1024$ pixels, and 512 slices were acquired. Assessment of bone volume at the treated site was carefully performed using the 3D Image calculator INFINITTXelis software. To assess the effect of threshold on the treated site, the degree of bone density was arbitrarily determined in ROI as low-density (threshold 1101-1600, blue color), middle-density (threshold 1601-2100, orange color), and high-density bone (threshold $>2101$, red color). For consistency, the same settings and thresholds were used for each analysis and applied to each sample in the study. Additionally, the whole calvaria were stained using TRAP solution.

\section{Evaluation of new bone formation in vivo}

The 5-week-old male ICR mice were divided into two experimental groups comprising five mice each: PBStreated (control) and recombinant protein of Cldn11treated mice. The mice received subcutaneous injections over the calvarial surface with or without Cldn11 (0.6 mg/ mouse) daily for 21 days. All mice were euthanized 21 days after treatment, and the dissected calvaria were fixed with $4 \%$ paraformaldehyde for 1 day. The calvaria were analyzed using high-resolution micro-CT to obtain 3D images. Assessment of bone volume at the treated site was performed as an analysis of the calvarial bone erosion model. The area of new bone that formed over the calvarial surface was quantified using Image-Pro Plus version 4.0.

\section{Histological analysis}

Calvarial bones were isolated and fixed with 10\% neutral-buffered formalin on day 1 and then decalcified with 12\% EDTA for 7 days. Decalcified bones were embedded in paraffin, cut to a thickness of $5 \mu \mathrm{m}$, and stained with hematoxylin and eosin to distinguish between nuclei and matrix. Other sections were stained with TRAP to visualize OCs. The number of OCs was determined using the histomorphometric results of the sections stained with TRAP. Stained bones were photographed under a light microscope.

\section{Statistical analysis}

Experiments were conducted at least three times, and the data were expressed as the mean \pm standard deviation (SD). All statistical analyses were performed using the Statistical Package for the Social Sciences Software (SPSS; Korean version 14.0). Student's $t$-test was used to compare the parameters between two groups, whereas the analysis of variance test followed by the Tukey's post hoc test was used to compare the parameters among three groups. $P<0.05$ was considered statistically significant.

\section{Results \\ $O C$ differentiation and function are suppressed by Cldn 11 overexpression without affecting the expression levels of OPG and RANKL}

To explore the role of Cldn11 in the interaction between OBs and OCs, we investigated the effect of Cldn11 on the expression of OPG and RANKL in OBs induced by IL-1 or both $\mathrm{PGE}_{2}$ and $\mathrm{Vit}_{3}$. Although the mRNA expression of $O P G$ and $R A N K L$ was significantly controlled by IL-1 or both $\mathrm{PGE}_{2}$ and $\mathrm{VitD}_{3}$ in the $\mathrm{OB}$ group transfected with pMX, Cldn11 overexpression did not exert any effects on the expression of these two cytokines (Fig. 1a). Indeed, under these conditions, Cldn11 had no ability to inhibit the formation of TRAPpositive OCs (Fig. 1b). Based on these results, we suggest that $O P G$ and $R A N K L$ are not the direct target genes of Cldn11 and that Cldn11 is not directly involved in OBmediated osteoclastogenesis. Hence, we then focused on the effects of Cldn 11 on OC differentiation and function under a microenvironment prepared by BMMs in the presence of M-CSF and RANKL. The expression of Cldn11 was induced on days 2 and 3 of RANKL treatment (Fig. 1c), and overexpression of Cldn11 significantly diminished the formation of $\mathrm{OCs}$ and the number of TRAP-positive MNCs (nuclei $>5$ ) derived from BMMs (Fig. 1d, e). We further postulated that Cldn11 has an ability to regulate osteoclastic bone resorption. To test this assumption, we generated mature OCs from bone marrow precursors by culturing them with $\mathrm{OBs}$, and retroviral transfection was performed using pMX or pMX-Cldn11. As shown in Fig. 1f, g, while numerous resorption pits were produced by mature OCs cultured on the top of hydroxyapatite-coated plates (Fig. If, middle and Fig. 1g, right) or dentin slices (Fig. 1f, right and Fig. $1 \mathrm{~g}$, right) in the pMX retroviral vector-transfected group, 


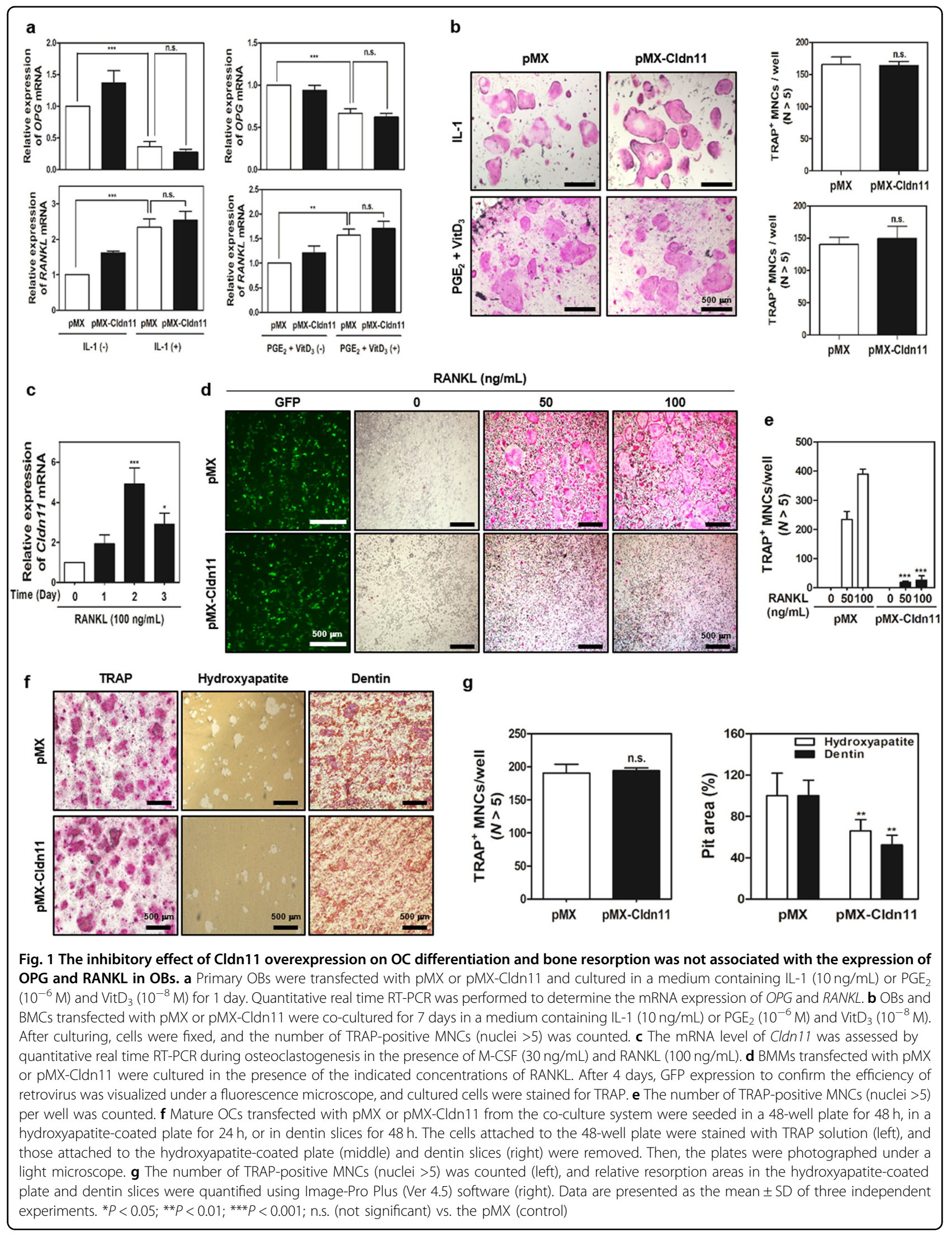




$$
\begin{aligned}
& \text { a }
\end{aligned}
$$

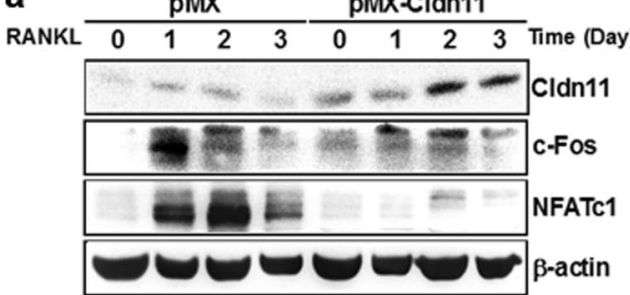

b RANKL

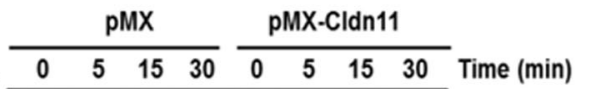

d
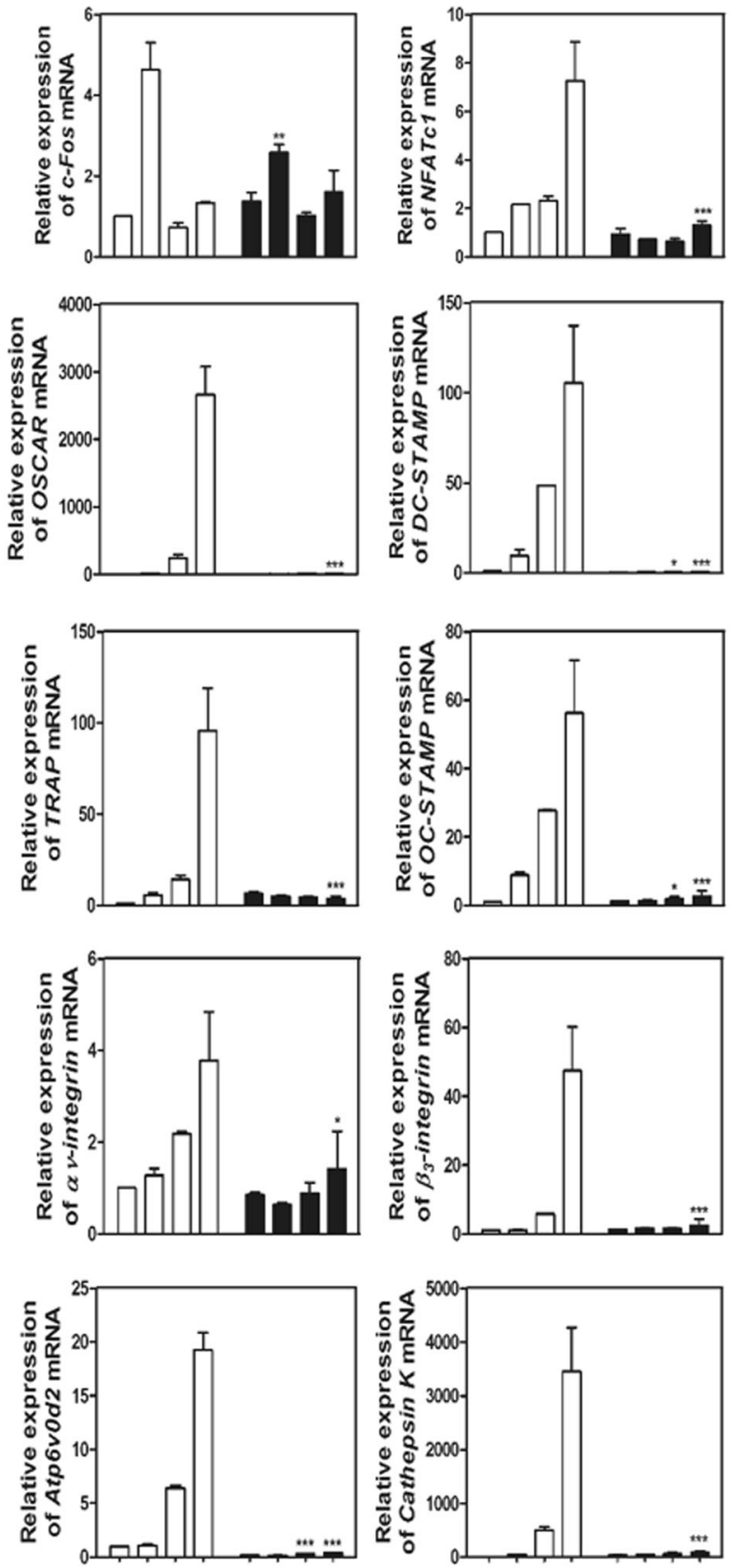

Time (Day) \begin{tabular}{rrrrrrrr}
0 & 1 & 2 & 3 \\
\hline
\end{tabular}

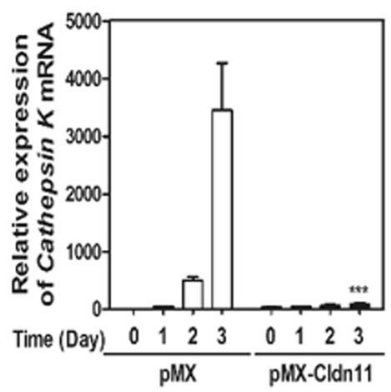

Fig. 2 Overexpression of Cldn11 reduces RANKL-induced osteoclastogenesis associated with signaling and marker genes. a-c BMMs were transduced with pMX or pMX-Cldn11 retrovirus and (a) then cultured in the presence of $\mathrm{M}$-CSF $(30 \mathrm{ng} / \mathrm{mL})$ and RANKL $(100 \mathrm{ng} / \mathrm{mL})$ for the indicated time. b, c Infected BMMs were serum starved for $6 \mathrm{~h}$ and stimulated with RANKL $(100 \mathrm{ng} / \mathrm{mL})$ for the indicated time points. Total cell lysates were analyzed by performing western blotting with the indicated antibodies. $\beta$-Actin was used as an internal control. $\mathbf{d}$ BMMs were transduced with pMX or pMX-Cldn11 retrovirus and cultured in the presence of $M-C S F(30 \mathrm{ng} / \mathrm{mL})$ and RANKL (100 ng/mL) for the indicated times. The mRNA expression of the indicated genes was analyzed by performing quantitative real time RT-PCR. Data are presented as the mean \pm SD of three independent experiments. ${ }^{*} P<0.05 ;{ }^{*} P<0.01 ;{ }^{* *} P<0.001$ vs. the $\mathrm{pMX}$ (control) of the indicated time point, respectively 


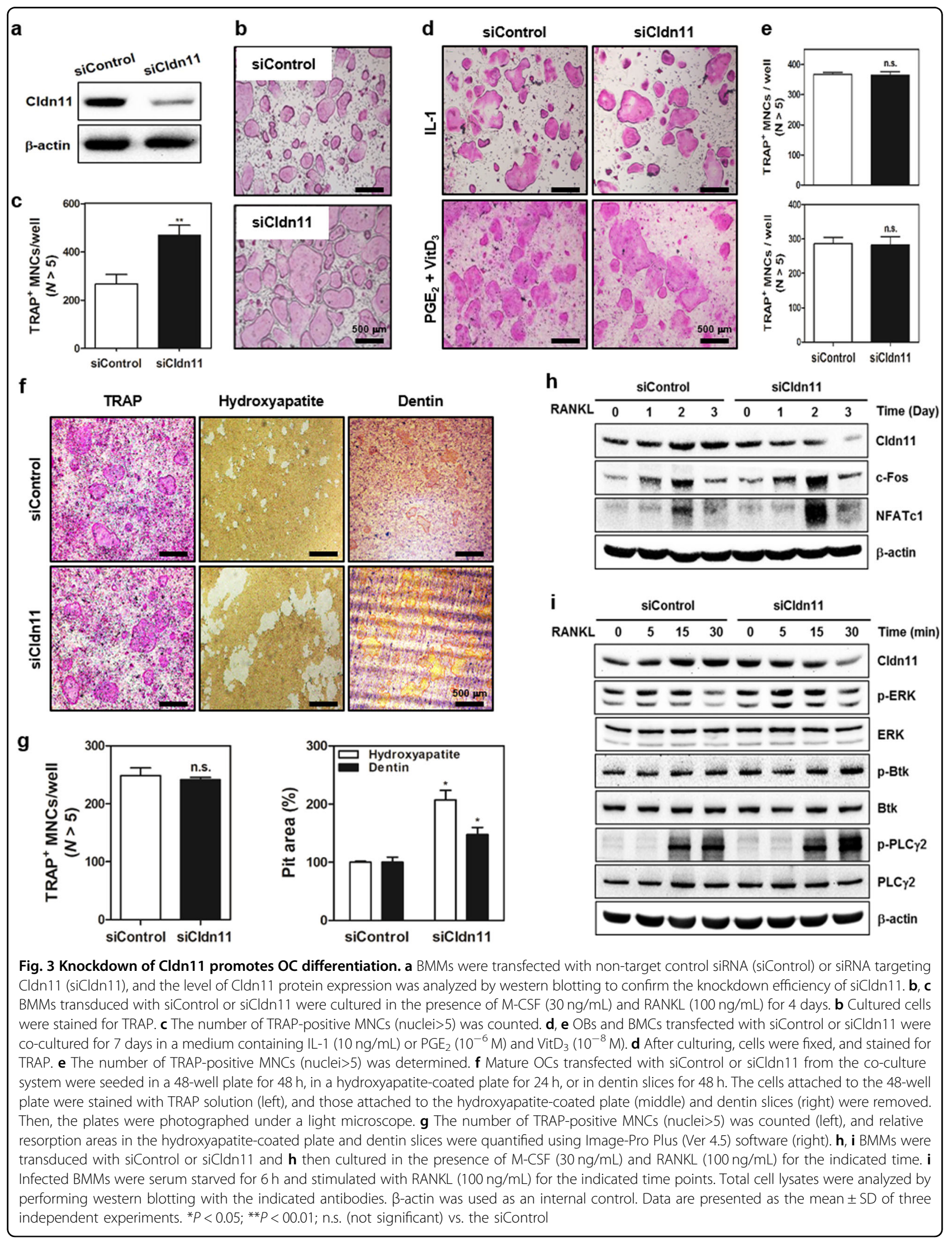


these resorption pits decreased with robust Cldn11 expression without any cytotoxicity (Fig. 1f, left and Fig. $1 \mathrm{~g}$, left). These data suggest that Cldn11 is a negative regulator of $\mathrm{OC}$ differentiation and that it functions independently of OPG and RANKL in OBs.

\section{Cldn 11 overexpression negatively regulates the RANKL-} induced expression of c-Fos, NFATc1, and OC marker genes and inhibits the RANKL-induced phosphorylation of ERK, Btk, and PLC 2

We performed western blotting and quantitative realtime RT-PCR to elucidate the molecular mechanism underlying the negative regulation of Cldn11 on RANKLinduced osteoclastogenesis. Compared with BMMs transduced with an empty vector, the mRNA and protein levels of two major transcription factors, c-Fos and NFATc1, and their upstream signal transducers, including ERK, Btk, and PLCY2, were downregulated in cultures overexpressing Cldn11 in response to RANKL (Fig. 2a, c; Figures S1a, c). However, the RANKL-induced phosphorylation of p38, Akt, JNK, and IKB was not affected by Cldn11 overexpression (Fig. 2b; Figures S1b). Negative regulation of Cldn11 on c-Fos and NFATc1 was further supported by quantitative real-time RT-PCR, and this potent inhibition contributed to the inhibition of expression of various $\mathrm{OC}$-associated marker genes, such as OC-associated receptor (OSCAR), TRAP, DC-STAMP, OC-STAMP, $\alpha v$-integrin, $\beta 3$-integrin, Atp6v0d2, and Cathepsin K (Fig. 2d). These results show that Cldn11 acts as a negative regulator in RANKL-mediated osteoclastogenesis via the de-phosphorylation of ERK, Btk, and $\mathrm{PLC} \gamma 2$, which is followed by the suppression of c-Fos and NFATc1, leading to decreased levels of OC-specific genes.

\section{Downregulation of Cldn11 enhances RANKL-induced osteoclastic activity}

Because Cldn11 acts as a negative regulator of osteoclastogenesis, we investigated its physiological role in osteoclastogenesis by using siRNAs. After confirming the siRNA-mediated knockdown efficiency of Cldn11 in BMMs (Fig. 3a), we first observed the accelerated formation of OCs and an increase in the number of TRAPpositive MNCs (nuclei $>5$ ) in the Cldn11 silencing group in comparison with the control (Fig. 3b, c). This relationship between Cldn11 and OC differentiation was not detected in the co-culture system of the control or in Cldn11 siRNA-transfected BMCs with OB in the presence of IL-1 or $\mathrm{PGE}_{2}$ and VitD ${ }_{3}$, which is in line with Fig. $1 \mathrm{~b}$ (Fig. 3d, e). However, knockdown of Cldn11 in mature OC derived from the co-culture model was found to increase bone-resorbing activity in hydroxyapatite-coated plates or dentin slices without affecting the survival of OCs (Fig. 3f, g). In terms of the molecular mechanisms, the silencing of Cldn11 in BMMs resulted in a significant increase in the induction of c-Fos and NFATc1 (Fig. 3h; Figures S2a) and the phosphorylation of ERK, Btk, and PLCY2 (Fig. 3i; Figures S2b). Taken together, these results suggest that Cldn11 silencing enhances osteoclastogenesis by increasing the phosphorylation of ERK, Btk, and PLC 2 , which is followed by the activation of c-Fos and NFATc1.

\section{Cldn 11 overexpression enhances $O B$ differentiation via Smad1/5/8, ERK, and JNK}

Based on this effect in osteoclastogenesis by gain of function and loss of function of Cldn11, we determined whether Cldn11 is also involved in osteoclastogenesis. As shown in Fig. 4a, elevated expression of Cldn11 was detected during $\mathrm{OB}$ differentiation induced by $\mathrm{AA}$ and $\beta$ gly, leading us to think that Cldn11 may have the ability to modulate osteogenesis. Thus, we tested the effect of Cldn11 on osteoclastogenesis using primary OBs ectopically expressed with pMX or pMX-Cldn11. Interestingly, Cldn11 overexpression promoted not only the formation of ALP and ARS-positive colonies, which are a marker of bone formation and calcium accumulation, but also their activity, and this enhancement was achieved via the upregulation of Smad1/5/8, ERK, and JNK, which are essential for the early stages of $\mathrm{OB}$ differentiation (Fig. 4b-d; Figures S3). Consistent with this enhancement, we observed that the expression levels of bone formation markers, including Runx2, ALP, osteocalcin (OCN), osteopontin $(O P N)$, type 1 collagen $\alpha($ Coll $\alpha)$, and bone sialoprotein $(B S P)$, were higher in the cultures overexpressing Cldn11 than in the vector-transfected control group (Fig. 4e). These findings indicate that Cldn11 exerts a positive role in osteoblastogenesis.

\section{Knockdown of Cldn11 attenuates osteoblastic activity}

After we observed that osteoblastogenesis was inhibited by Cldn 11 overexpression, we then sought to assess the effect of Cldn11 silencing on OB differentiation. To test this effect, we cultured primary $\mathrm{OB}$ precursor cells transfected with control siRNA or Cldn11-specific siRNA in the presence of osteogenic factors. As expected, we also verified that OBs transfected with siRNA targeting Cldn11 were defective in ALP and ARS activity (Fig. 5a and b), and this impairment contributed to the downregulation of Smad1/5/8, ERK, and JNK phosphorylation in the early stage of OB differentiation (Fig. 5c; Figures S4). These data reconfirm that the effect of Cldn11 on OB activity was accompanied by the regulation of Smad1/5/8, ERK, and JNK.

\section{Cldn11 regulates osteoclastogenesis by interacting with EphrinB2 ligand}

To determine whether bidirectional signaling dependent on the EphrinB2 ligand and EphB4 receptor is 
a

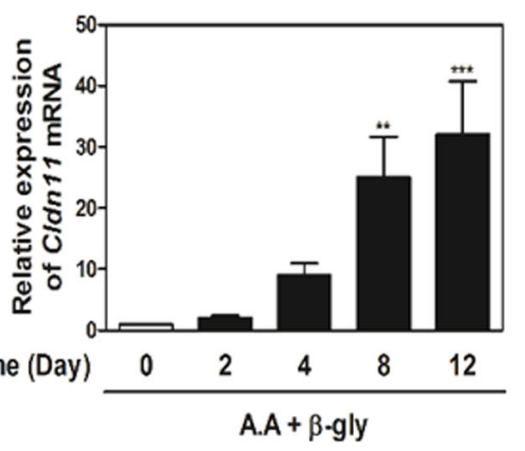

b
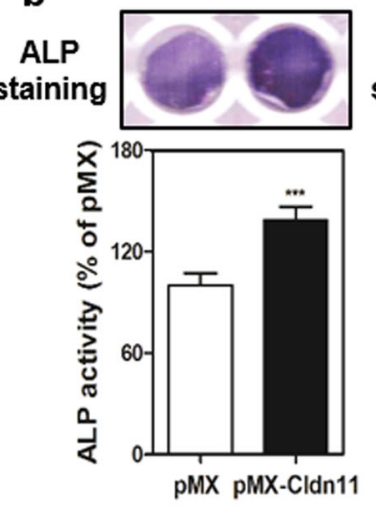

e
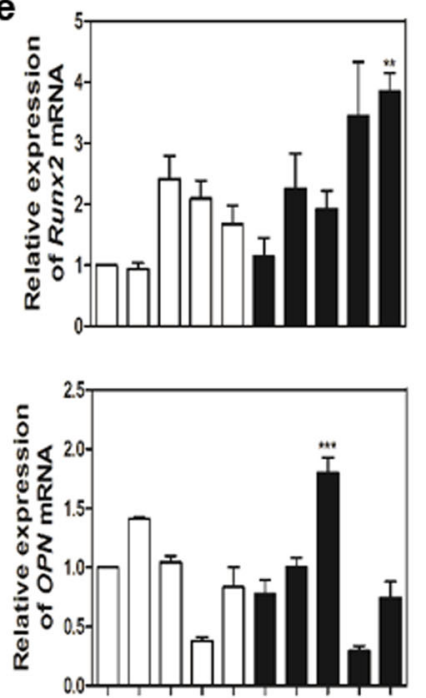

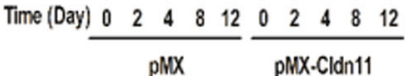

C ARS staining
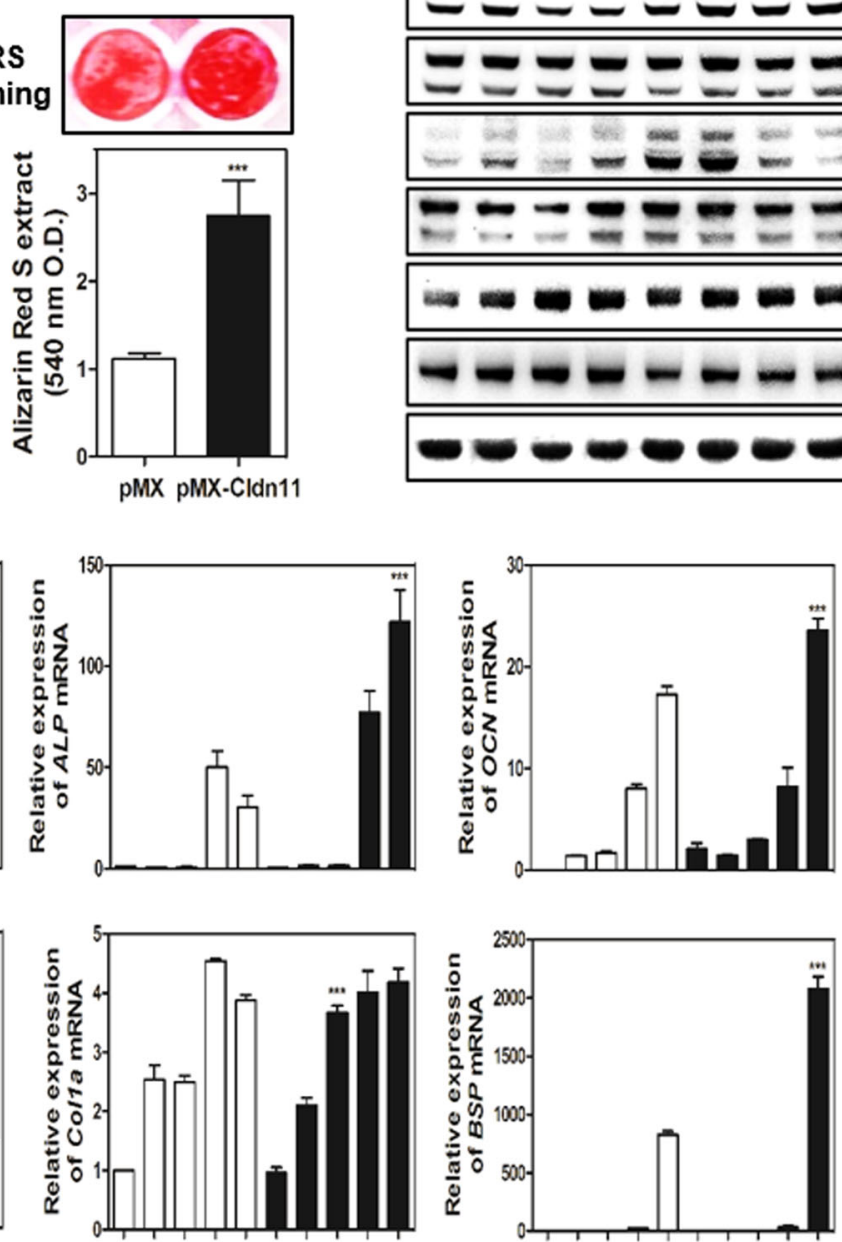

Time (Day) $0 \begin{array}{llllllllllllll}0 & 2 & 8 & 12 & 0 & 2 & 4 & 8 & 12\end{array}$

PMX-Cldn11

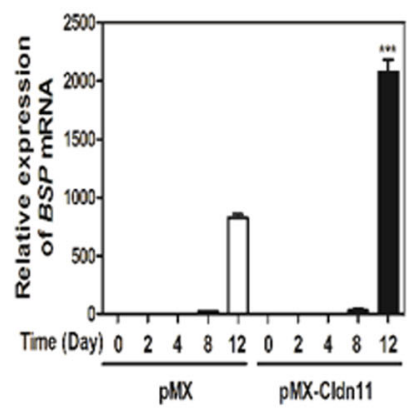

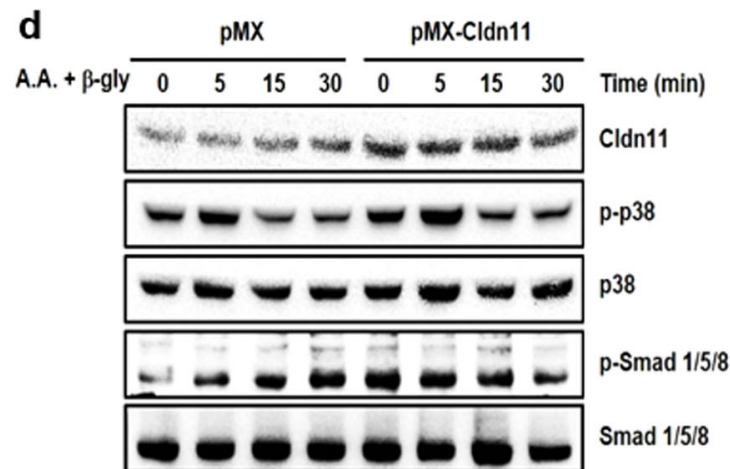

ニニニニここニこ p.ERK

$=-0=0-0$ ERK

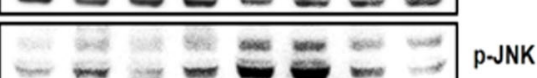

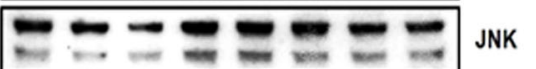

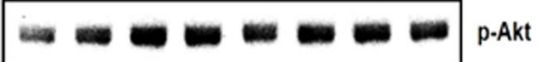

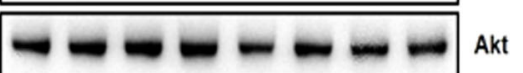

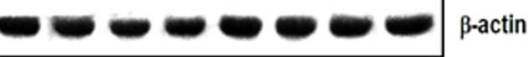

Fig. 4 Overexpression of Cldn11 enhances $O B$ differentiation and its intracellular signaling. a-e Primary OB precursor cells were cultured in osteogenic medium containing AA $(50 \mu \mathrm{g} / \mathrm{mL})$ and $\beta$-gly $(10 \mathrm{mM})$. a Cells were cultured for the indicated times, and mRNA levels of Cldn 11 were analyzed by quantitative real-time RT-PCR. $\mathbf{b}$ OBs transfected with pMX or pMX-Cldn11 were cultured for 7 days in osteoblastogenic medium. ALPpositive cells were monitored by ALP staining, and ALP activity was expressed a function of released $p$-nitrophenol. c Calcium deposits for matrix mineralization were measured by ARS staining after 21 days, and the intensity of staining was quantified with CPC. $\mathbf{d}$ Total cell lysates from OBs transfected with $\mathrm{pMX}$ or pMX-Cldn11 were analyzed by performing western blotting with the indicated antibodies. $\beta$-actin was used as an internal control. e Cells were cultured for the indicated times, and the mRNA expression of the indicated genes was analyzed by performing quantitative real time RT-PCR. Data are presented as the mean \pm SD of three independent experiments. ${ }^{* *} P<0.01$; ${ }^{* * *} P<0.001 \mathrm{vs}$. pMX (control) of the indicated time point, respectively 


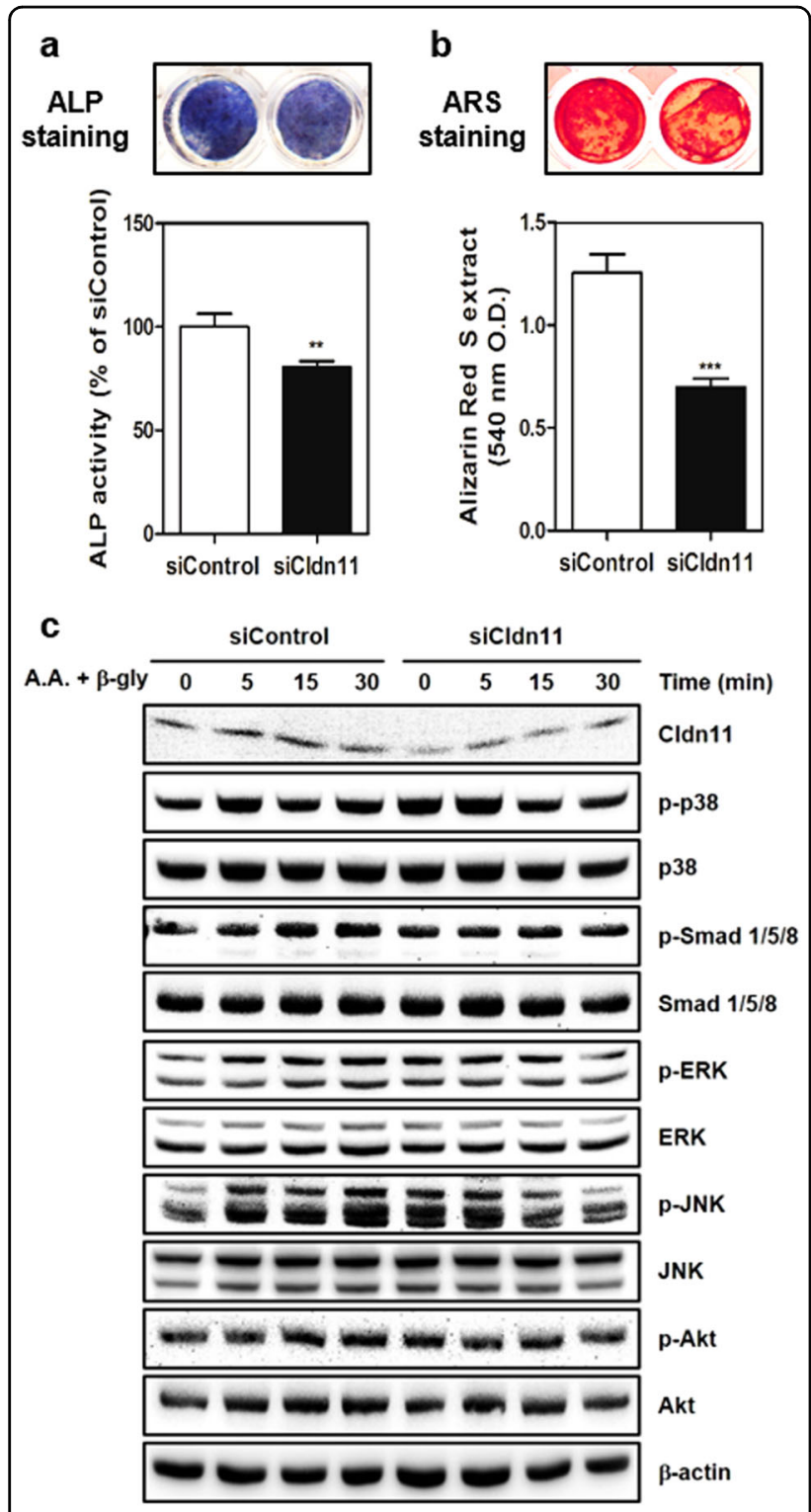

Fig. 5 Knockdown of Cldn 11 suppresses OB differentiation. a OBs transfected with siControl or siCldn11 were cultured for 7 days in osteoblastogenic medium. ALP-positive cells were monitored by ALP staining, and ALP activity was expressed as a function of released $p$ nitrophenol. b Calcium deposits for matrix mineralization were measured by ARS staining after 21 days, and the intensity of staining was quantified with CPC. c Total cell lysates from OBs transfected with siControl or siCldn11 were analyzed by carrying out western blotting with the indicated antibodies. $\beta$-actin was used as an internal control. Data are presented as the mean \pm SD of three independent experiments. ${ }^{*} P<0.01 ;{ }^{* *} P<0.001$ vs. the siControl

involved in the negative feedback role of Cldn11 on OC differentiation, we evaluated the relationship between Cldn11 and the EphrinB2/EphB4 axis. As shown in Fig. 6a, b, we found that EphrinB2 or EphB4 was colocalized with Cldn11 in the membranes of OC precursor cells and that the mRNA expression of EphrinB2 or $E p h B 4$ was induced in a time-dependent manner during OC differentiation. To verify whether Cldn11 interacted with EphrinB2 or EphB4, we performed coimmunoprecipitation (co-IP) experiments on OC wholecell extracts using EphrinB2 or EphB4 antibodies. We confirmed that Cldn11, EphrinB2, and EphB4 were expressed on OCs and that Cldn11 selectively interacted with EphrinB2 but not EphB4 (Fig. 6c). Furthermore, the degree of this interaction was attenuated in the Cldn11 silencing group compared with the interaction in the control siRNA group at $24 \mathrm{~h}$ after RANKL treatment (Fig. 6d). Reflecting on this connection between Cldn11 and EphrinB2, we hypothesized that Cldn11 may regulate EphrinB2 reverse signaling into OCs. To address this hypothesis, we added soluble chimeric EphB4-Fc to activate EphrinB2-dependent reverse signaling in $\mathrm{OC}$ cultures. Consequently, the abnormally accelerated formation of TRAP-positive OCs (Fig. 6e) and pit areas on dentin slices (Fig. 6f) derived from siRNA-induced knockdown of Cldn11 was sufficiently overcome by EphB4 treatment. These results suggest that Cldn11 negatively controls $\mathrm{OC}$ function via interacting with EphrinB2.

\section{Cldn11 modulates osteoblastogenesis by interacting with EphB4 receptor}

We next set out to investigate whether Cldn11 interacted with EphrinB2 or EphB4 in OBs. As shown Fig. 7a, b, we found that Cldn11 was co-localized with EphrinB2 or EphB4 in the membranes of OBs (Fig. 7a) and that the mRNA expression of EphrinB2 or EphB4 increased in a time-dependent manner during $\mathrm{OB}$ differentiation (Fig. 7b). However, our results revealed that Cldn 11 only distinctly interacted with EphB4, not EphrinB2, in OBs by co-IP assay (Fig. 7c). Additionally, the degree of interaction between the two molecules was attenuated in the Cldn11 silencing group compared with the degree of interaction in the control siRNA group at 2 and 4 days after AA and $\beta$-gly treatment (Fig. 7d). Moreover, the impaired OB differentiation induced by Cldn11 siRNAs was significantly reversed through treatment with EphrinB2-Fc, which activates EphB4-dependent forward signaling into OBs (Fig. 7e, f). Taken together, these findings indicate that Cldn11 positively mediates osteoblastogenesis activity by relating with EphB4.

\section{Local injection of Cldn11 recombinant protein protects LPS-induced erosion of calvarial bone areas and induces new bone formation}

Based on the in vitro effects of Cldn11 on osteoclastic bone resorption and osteoblastic bone formation, we evaluated the effect of locally injected Cldn 11 recombinant protein onto mouse calvaria with the expectation of 

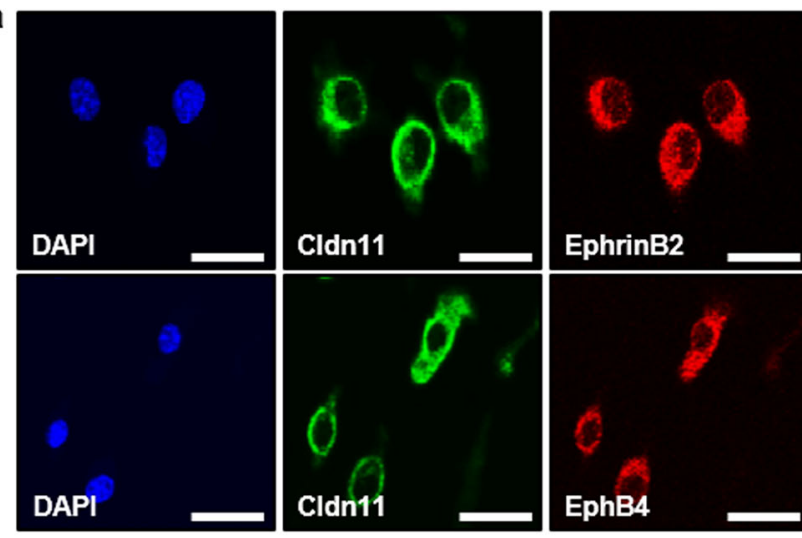

b

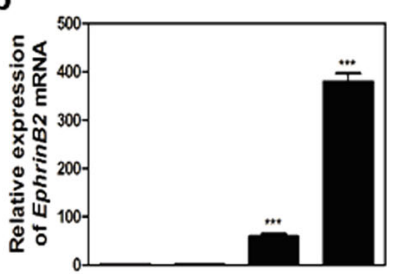

c
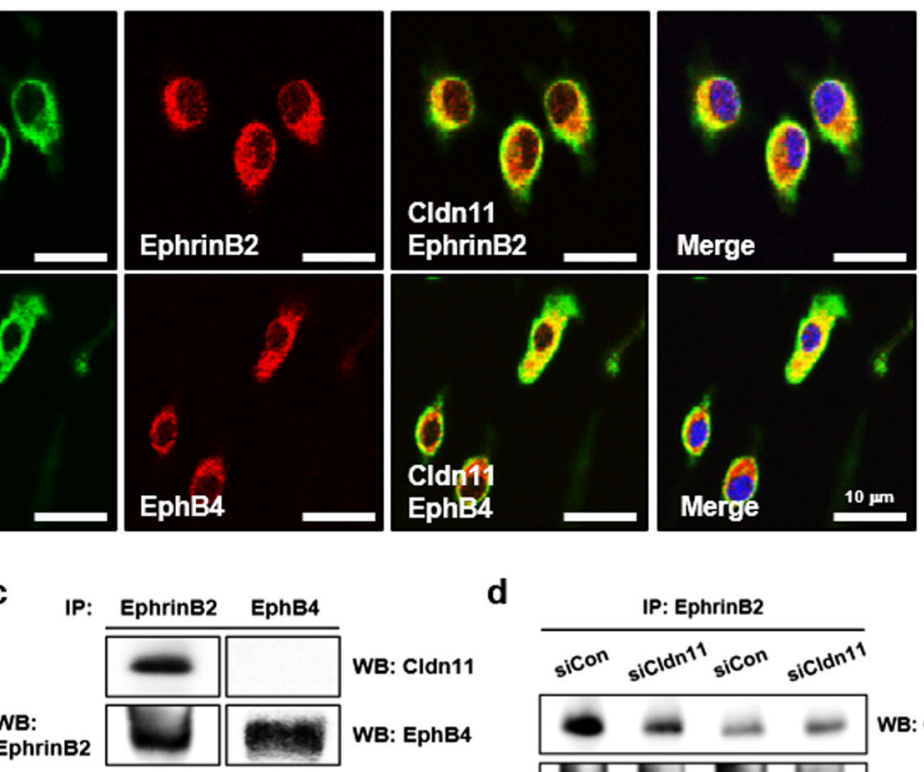

d
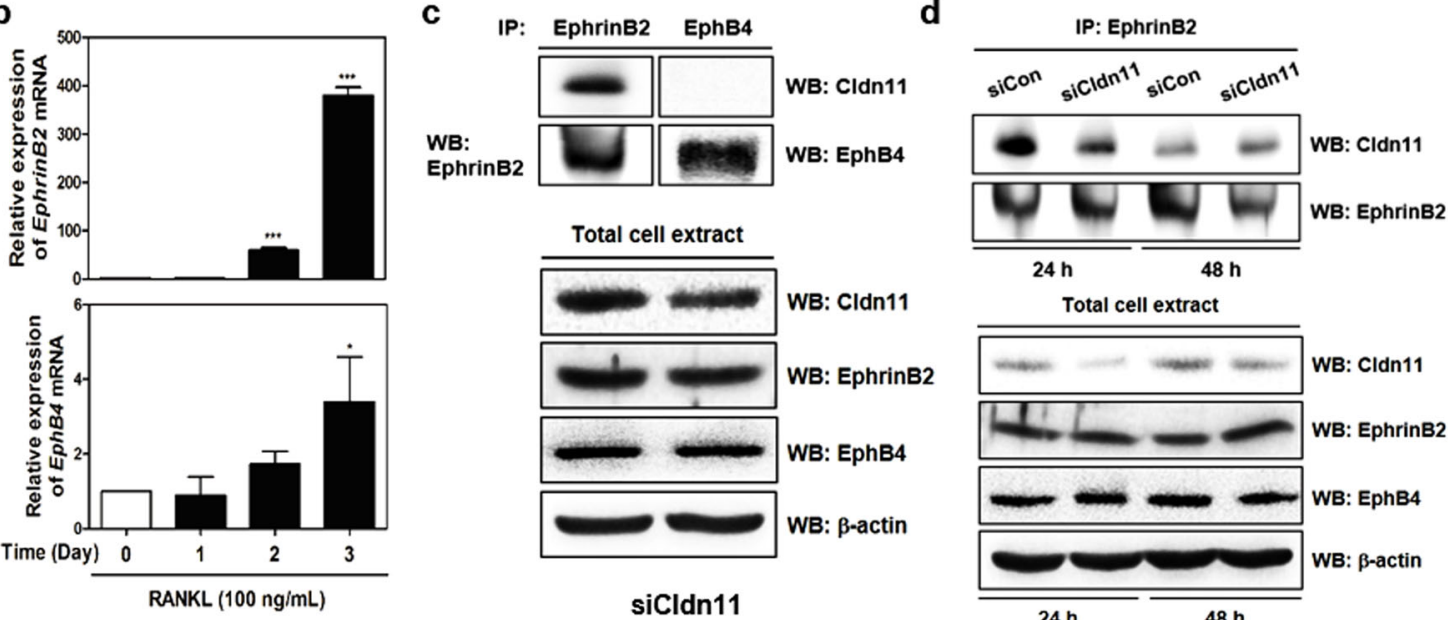

e

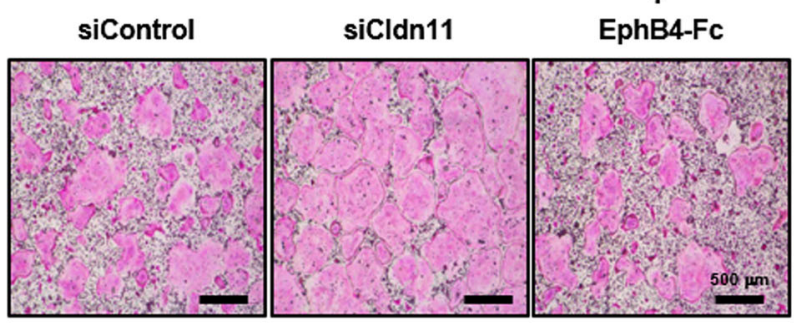

siCldn11

f
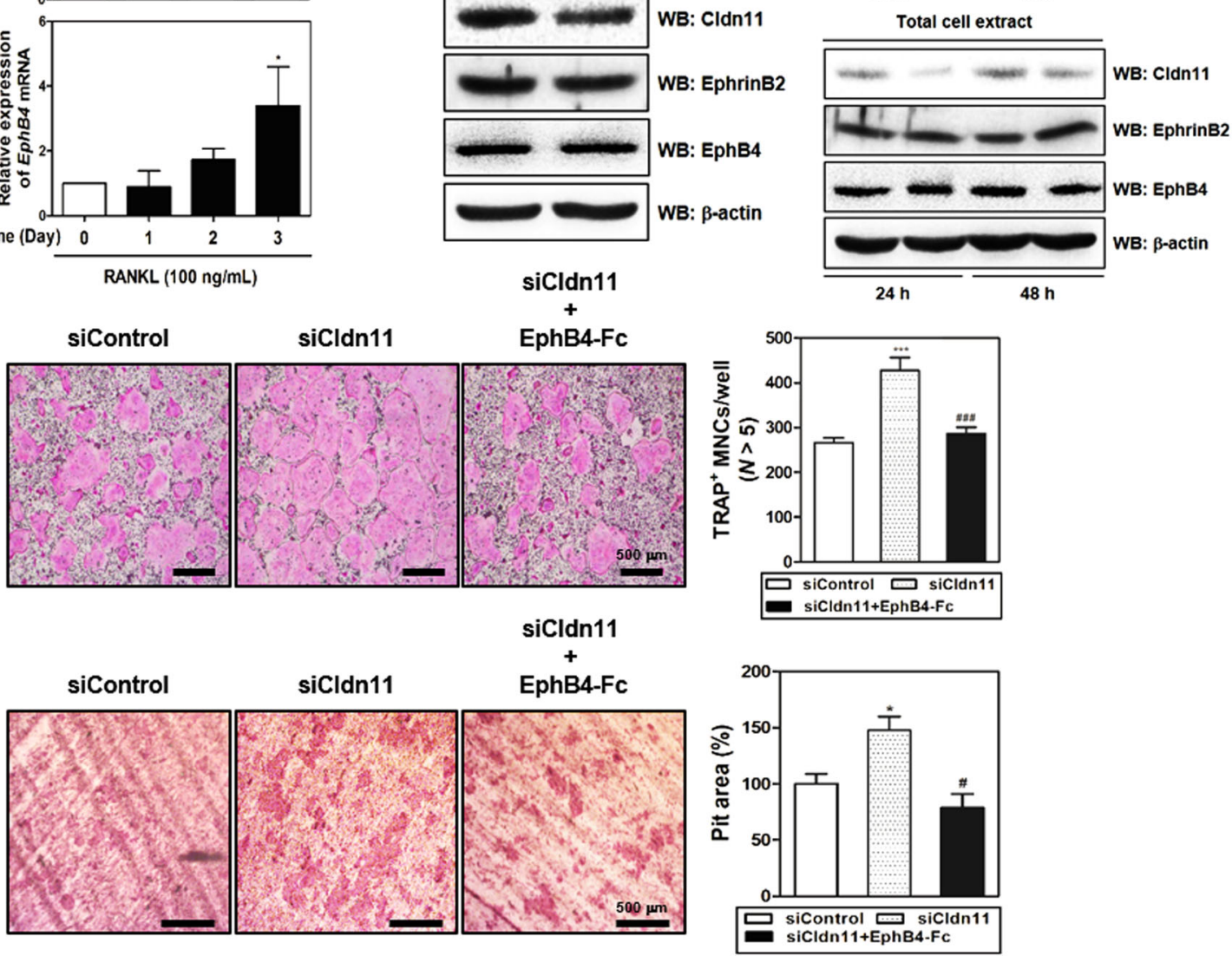

Fig. 6 (See legend on next page.) 
(see figure on previous page)

Fig. 6 Cldn11 negatively modulates osteoclastogenesis via interaction with the EphrinB2 ligand. a The expression of Cldn11, EphrinB2, and EphB4 and the co-localization of these proteins in osteoclast precursor cells were observed with a confocal imaging assay. $\mathbf{b}$ The mRNA expression of EphrinB2 and EphB4 was evaluated during preOC differentiation into mature OCs by quantitative RT-PCR. c BMMs were cultured in the presence of MCSF $(10 \mathrm{ng} / \mathrm{mL})$ for 1 day prior to cell lysis. Lysates were immunoprecipitated with EphrinB2 or EphB4 antibody and subsequently immunoblotted with Cldn11 antibody. d BMMs transfected with siControl or siCldn11 were cultured and lysed in the presence of M-CSF (30 ng/mL) and RANKL (100 $\mathrm{ng} / \mathrm{mL}$ ) for 24 and $48 \mathrm{~h}$. Lysates were immunoprecipitated with EphrinB2 antibody and subsequently immunoblotted with Cldn 11 antibody. c, d Coimmunoprecipitated samples (top) or total cell extracts (bottom) were subjected to western blotting to detect Cldn11, EphrinB2, EphB4, and $\beta$-actin. e BMMs were transfected with control siRNA or siCldn11, and one of the siCldn11 groups was treated with EphB4-Fc. Subsequently, the cells were cultured in the presence of M-CSF $(30 \mathrm{ng} / \mathrm{mL})$ and RANKL $(100 \mathrm{ng} / \mathrm{mL})$ for 4 days. After culturing, cells were fixed and stained (left), and the number of TRAP-positive MNCS was counted (right). (f) Mature OCs transfected with siControl or siCldn11 from the co-culture system were seeded on dentin slices, and one of the siCldn 11 groups was treated with EphB4-Fc, and then mature OCs were cultured for $48 \mathrm{~h}$. Attached cells on the plates were removed and photographed under a light microscope (left). Pit areas were quantified using Image-Pro Plus (Ver 4.5) software (right). ${ }^{*} P<0.05$; ${ }^{* * *} P<$

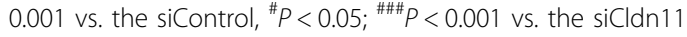

dual anti-resorptive and anabolic action in vivo. As shown in Fig. 8a, the increased TRAP-positive regions of whole calvaria in the LPS injection group compared with that of the PBS-treated control were significantly prevented through treatment with Cldn11 recombinant protein. Indeed, the results obtained using bone histomorphometric analysis after micro-CT and histology of calvarial sections revealed that local injection of Cldn11 recombinant protein altered the impaired bone density of calvaria mediated by LPS and markedly recovered excessive erosion of bone surface and formation of $\mathrm{OC}$ per field in calvarial regions (Fig. 8b-d). Next, in the results of the evaluation of the anabolic effect of Cldn11 recombinant protein, although there was a slight increase in bone density in the calvaria of normal mice locally injected with Cldn11 recombinant protein (Fig. 8e), we detected new areas of bone growth in calvaria sections of Cldn11treated mice compared with the sections of the control group (Fig. 8f). Together, these in vivo results demonstrate the anti-resorptive and anabolic properties of Cldn11 injection in the bone microenvironment.

\section{Discussion}

Forced or silenced Cldn11 expression in cancer cells affects motility and invasiveness, indicating that Cldn11 may have a therapeutic role in preventing cancer progression $^{19-21}$. Detection of promoter hypermethylation of Cldn11 can be used as a diagnostic parameter for distinguishing malignant melanoma from benign melanocytic lesions $^{22,23}$. Importantly, the indirect effect of Cldn11 on cancer progression of breast tumor cells has been previously documented, motivating us to clarify the role of Cldn11 in bone homeostasis ${ }^{24-26}$. We therefore speculate that Cldn11 has the capacity to act as a biomarker for impaired bone mass-related metabolic disorders.

Given the dual anabolic-anti-resorptive action of Cldn11 defined in our experiments, we measured the effects of Cldn11 on the ratio of OPG/RANKL, which is a critical index for the steady state of bone mass and integrity to expand our understanding of Cldn11dependent regulatory pathways in bone metabolism ${ }^{27-30}$. Unexpectedly, in our study, genetic manipulation of Cldn11 did not mediate the mRNA levels of OPG or RANKL (Fig. 1a), leading us to think that other molecular targets aside from OPG and RANKL are correlated with Cldn11 in the bone remodeling process.

We examined previous evidence to identify the putative interacting partners of Cldn11 in bone metabolism and chose the transmembrane ligands, ephrins, and their tyrosine kinase receptor, Eph, as potential candidates. Although a direct association between $\mathrm{Cldn} 11$ and ephrins has not yet been established, several experimental studies in the field of molecular biology have proven that other Cldn proteins, including Cldn1 and Cldn4, interact with ephrin ligands and Eph receptors ${ }^{31-35}$. We found that EphrinB2 and its receptor, EphB4, selectively interacted with Cldn11 in OCs and OBs (Figs. 6c and 7c). Previously, the role of bidirectional signal transduction between EphrinB2 and EphB4 in bone metabolism was reported. Cell-cell communication between OCs and OBs is controlled through signaling along the EphrinB2/EphB4 axis, thereby maintaining balance in the activity of these two bone cells and contributing to the maintenance of bone homeostasis ${ }^{36}$. In this regard, activation of the EphrinB2 ligand located within the membrane of OCs is mediated by the EphB4 receptor secreted by OBs, impeding OC differentiation via the c-Fos-NFATc1 cascade, which is called "reverse signaling," and this negative feedback loop is achieved through the C-terminal YKV motif of EphrinB2, which is a binding site for PDZ (postsynaptic density protein, disks large, and zona occludens) domain proteins. On the other hand, activation of the EphB4 receptor located within OBs is stimulated by the EphrinB2 ligand produced by OCs, elevating $\mathrm{OB}$ differentiation, which is referred to as "forward signaling," and this positive regulation requires activated ERK1/2 pathways and attenuates RhoA activity ${ }^{36-38}$. In the present study, we confirmed the potential link of 


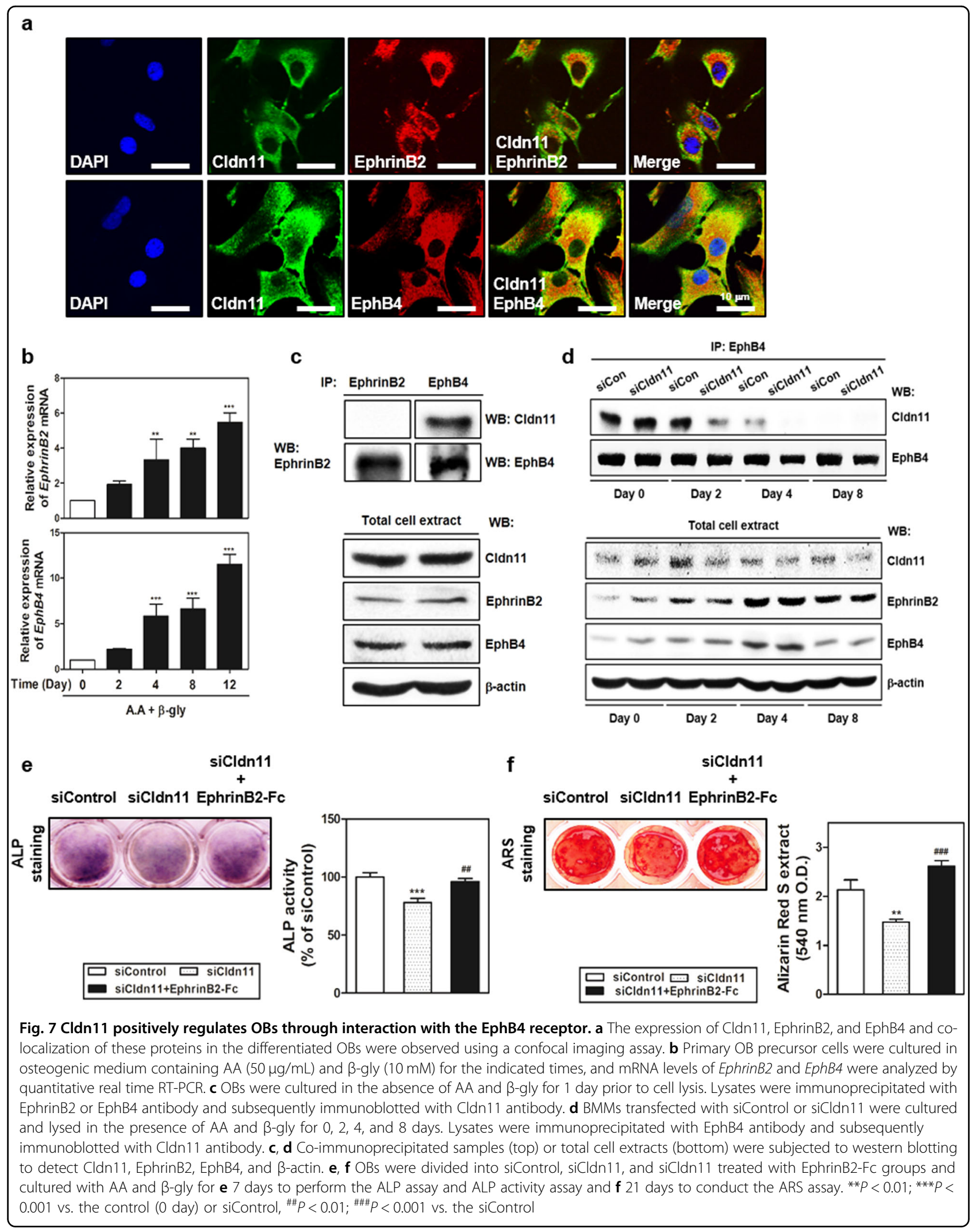




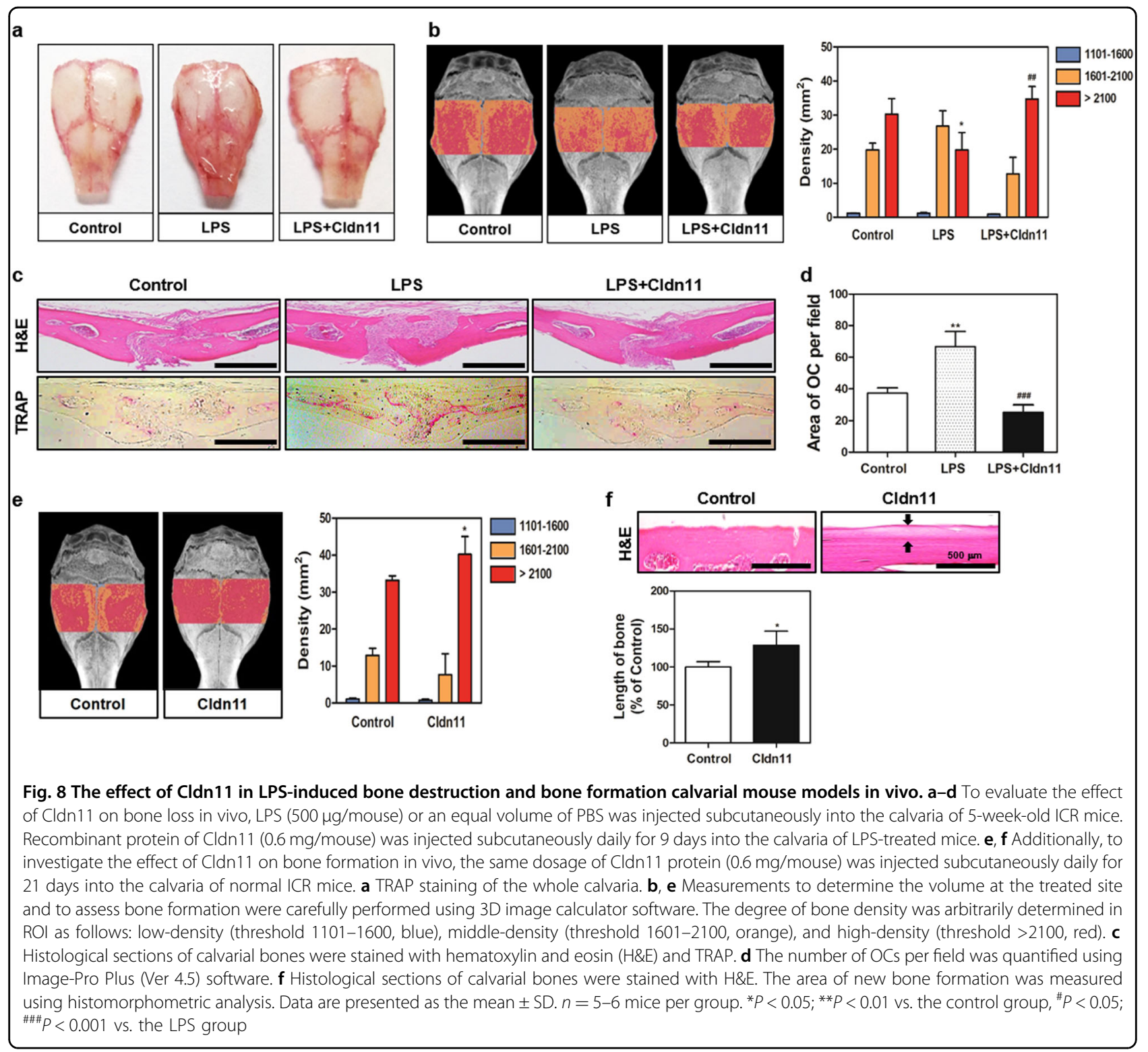

Cldn11 with both the EphrinB2 ligand and EphB4 receptor by proving the co-localization of Cldn11 with EphrinB2 and EphB4 in the membranes of OCs and OBs, respectively (Figs. 6a and 7a). Furthermore, to determine whether Cldn11 regulates the bidirectional signaling of EphrinB2/EphB4 within OC and OB lineage cells, we used recombinant mouse EphrinB2-Fc and EphB4-Fc to activate EphB4-dependent forward signaling and EphrinB2dependent reverse signaling in vitro. Notably, treatment of EphB4-Fc and EphrinB2-Fc in OC and OB culture was sufficient to reverse augmented $O C$ differentiation and bone-resorbing activity and impaired $\mathrm{OB}$ differentiation and mineral accumulation in response to siRNA-mediated knockdown of Cldn11 by activating EphrinB2 reverse signaling in OCs and EphB4 forward signaling in OBs
(Figs. $6 \mathrm{~d}-\mathrm{f}$ and $7 \mathrm{~d}, \mathrm{e}$ ), indicating that the negative and positive feedback modes of Cldn11 in OCs and OBs arose via the activation of reverse signaling in OCs through coupling with EphrinB2 and the stimulation of forward signaling in OBs in combination with EphB4. Based on these results, we speculated that Cldn11 regulates bone metabolism through bidirectional signaling of EphrinB2 and EphB4 by acting like an EphB4 receptor in OC differentiation in order to induce EphrinB2-mediated reverse signaling and by acting like an EphrinB2 ligand in $\mathrm{OB}$ differentiation in order to activate EphB4-mediated forward signaling.

However, further examination is necessary to characterize the precise mechanisms underlying the involvement of the EphrinB2/EphB4 axis in Cldn11-mediated 
anti-resorptive and anabolic events because the specific binding regions (domain or motif) of Cldn11 that recognize EphrinB2 and EphB4 have not been identified. The results of such an analysis may be used to better clarify the dual action of Cldn11 in bone mass homeostasis. Moreover, an unexpected finding was observed in our study. As shown in Fig. 6b, the pattern of EphB4 mRNA expression in BMMs slightly increased upon RANKL treatment in a time-dependent manner, which is in disagreement with the reported findings indicating that none of the EphB receptors (B1-B6) are detected in the same culture conditions $^{36}$. It is likely that weak expression of EphB4 (approximately 3.4-fold enhancement), as seen in our experiments, may have barely been detected with the gelbased RT-PCR analysis used by Zhao et $\mathrm{al}^{36}$.

Finally, to expand on the current in vitro analysis based on assessment of the role of Cldn11 on two key cells in the bone microenvironment, we conducted in vivo studies using a mouse calvarial model under pathologic and normal states. As a consequence, local subcutaneous injection of Cldn11 recombinant protein altered the osteoporotic bone phenotype that results from an acute inflammation response (Fig. 8a-d). In agreement with this phenomenon, treatment with siRNA of Cldn11 reversely led to the induction of more severe bone erosion phenotypes than those seen in the only LPS injection group

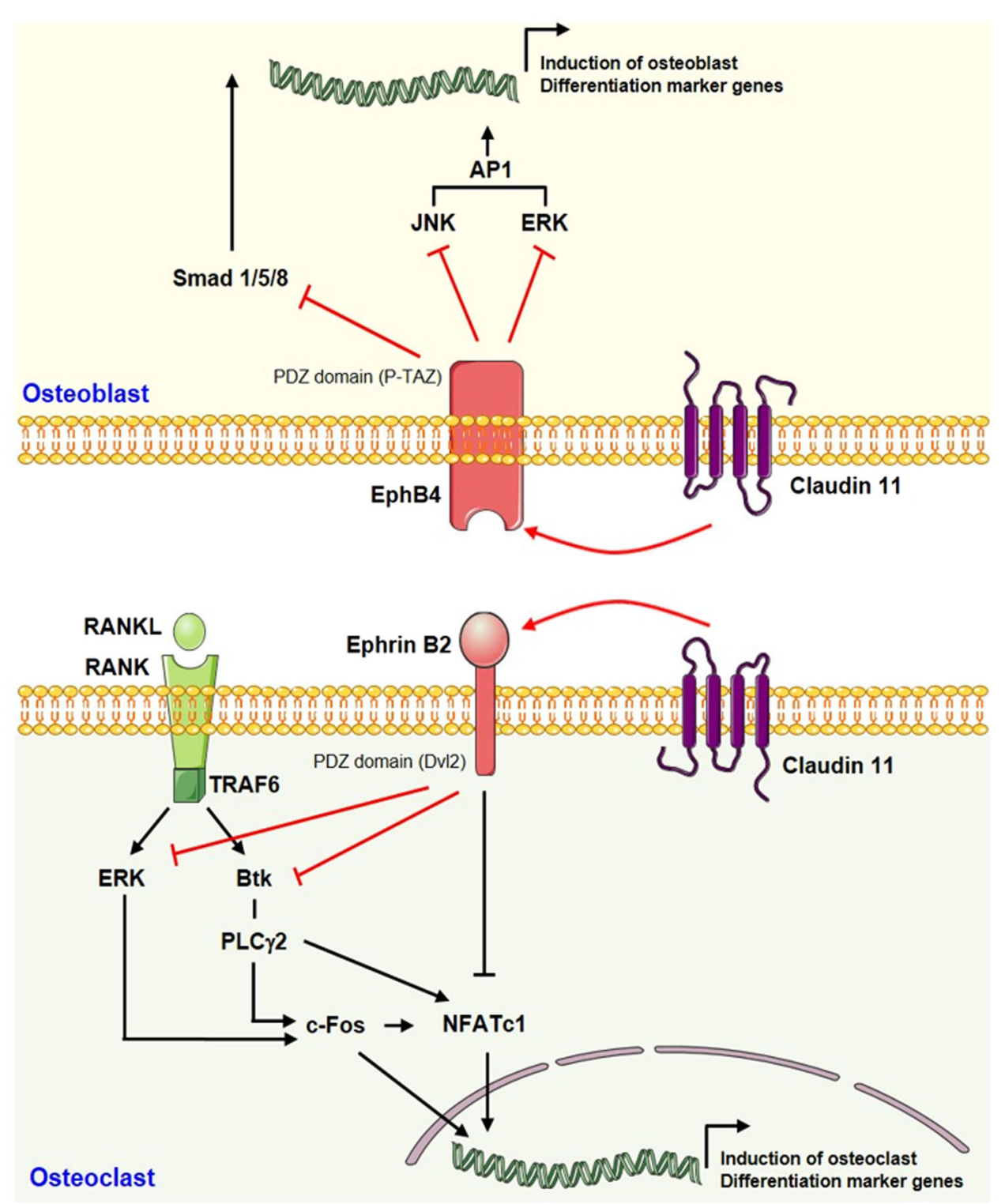

Fig. 9 A schematic diagram of the mechanism of Cldn11 in regulating bone homeostasis via bidrectional EphB4-EphrinB2 signaling. Cldn11-dependent dual event in bone metabolism arose from targeting EphrinB2 ligand reverse signaling into OC and EphB4 receptor forward signaling into $\mathrm{OB}$ 
(Figure S5). Under normal conditions, Cldn11-mediated formation of new bone is observed in calvarial histologic sections, whereas only a slight tendency for enhanced bone density was observed in the micro-CT experiment (Fig. 8e-g). Although this slightly increased rate of bone growth without any side effects needs additional investigation, we speculated that the significant extent of new bone formation takes a much longer period of investigation than 21 days.

In conclusion, we determined a previously unidentified role of Cldn11 in bone homeostasis and provided valuable insights into the Cldn11-mediated regulation of $\mathrm{OC}$ and OB differentiation (Fig. 9), highlighting that Cldn11 is a potential novel biomarker for the diagnosis and management of metabolic disorders characterized by low bone mass, such as osteoporosis.

\section{Acknowledgements}

This study was supported by a grant from the Basic Science Research Program through the National Research Foundation of Korea (NRF) funded by the Ministry of Education (NRF-2013R1A1A2059831 and NRF-

2015R1A2A2A01005899)

\section{Author details}

'Department of Anatomy, School of Medicine, Wonkwang University, Iksan, Republic of Korea. ${ }^{2}$ Medical Convergence Research Center, Wonkwang University Hospital, Iksan, Republic of Korea. ${ }^{3}$ Department of Radiology, School of Medicine, Wonkwang University, Iksan, Republic of Korea. ${ }^{4}$ Department of Internal Medicine, Division of Rheumatology, Wonkwang University, Iksan, Republic of Korea

\section{Conflict of interest}

The authors declare that they have no conflict of interest.

\section{Publisher's note}

Springer Nature remains neutral with regard to jurisdictional claims in published maps and institutional affiliations.

Supplementary information accompanies this paper at https://doi.org/ 10.1038/s12276-018-0076-3.

Received: 21 August 2017 Revised: 6 February 2018 Accepted: 23 February 2018.

Published online: 27 April 2018

\section{References}

1. Elkouby-Naor, L. \& Ben-Yosef, T. Functions of claudin tight junction proteins and their complex interactions in various physiological systems. Int. Rev. Cell Mol. Biol. 279, 1-32 (2010).

2. Schneeberger, E. E. \& Lynch, R. D. The tight junctions: a multifunctional complex. Am. J. Physiol. Cell Physiol. 286, C1213-C1228 (2004).

3. Matter, K. \& Balda, M. S. Signaling to and from tight junctions. Nat. Rev. Mol. Cell. Biol. 4, 225-236 (2003).

4. Tsukita, S., Furuse, M. \& Itoh, M. Multifunctional strands in tight junctions. Nat. Rev. Mol. Cell. Biol. 2, 285-293 (2001).

5. Günzel, D. \& Yu, A. S. Claudins and the modulation of tight junction permeability. Physiol. Rev. 93, 525-569 (2013).

6. Gow, A. et al. CNS myelin and sertoli cell tight junction strands are absent in Osp/claudin-11 null mice. Cell 99, 649-659 (1999).

7. Gow, A. et al. Deafness in Claudin 11-null mice reveals the critical contribution of basal cell tight junctions to stria vascularis function. J. Neurosci. 24, 7051-7062 (2004)
8. Kitajiri, S. et al. Compartmentalization established by claudin-11-based tight junctions in stria vascularis is required for hearing through generation of endocochlear potential. J. Cell Sci. 117, 5087-5096 (2004).

9. Shimobaba, S. et al. Claudin-18 inhibits cell proliferation and motility mediated by inhibition of phosphorylation of PDK1 and Akt in human lung adenocarcinoma A549 cells. Biochim. Biophys. Acta 1863, 1170-1178 (2016).

10. Suh, Y. et al. Claudin-1 induces epithelial-mesenchymal transition through activation of the c-Abl-ERK signaling pathway in human liver cells. Oncogene 32, 4873-4882 (2013).

11. Linares, G. R. et al. Claudin 18 is a novel negative regulator of bone resorption and osteoclast differentiation. J. Bone Miner. Res. 27, 1553-1565 (2012).

12. Lacey, D. L. et al. Osteoprotegerin ligand is a cytokine that regulates osteoclast differentiation and activation. Cell 93, 165-176 (1998).

13. Yasuda, H. et al. Osteoclast differentiation factor is a ligand for osteoprotegerin/osteoclastogenesis-inhibitory factor and is identical to TRANCE/RANKL. Proc. Natl. Acad. Sci. USA 95, 3597-3602 (1998).

14. Alshbool, F. Z. \& Mohan, S. Differential expression of claudin family members during osteoblast and osteoclast differentiation: Cldn-1 is a novel positive regulator of osteoblastogenesis. PLOS ONE 9, e114357 (2014).

15. Baek, J. M., Kim, J. Y., Yoon, K. H., Oh, J. \& Lee, M. S. Ebselen is a potential antiosteoporosis agent by suppressing receptor activator of nuclear factor kappaB ligand-induced osteoclast differentiation in vitro and lipopolysaccharideinduced inflammatory bone destruction in vivo. Int J. Biol. Sci. 12, 478-488 (2016).

16. Kim, J. Y. et al. Emodin regulates bone remodeling by inhibiting osteoclastogenesis and stimulating osteoblast formation. J. Bone Miner. Res. 29 1541-1553 (2014)

17. Baek, J. M. et al. Esculetin attenuates receptor activator of nuclear factor kappaB ligand-mediated osteoclast differentiation through c-Fos/nuclear factor of activated T-cells C1 signaling pathway. Biochem. Biophys. Res. Commun. 461, 334-341 (2015).

18. Kim, J. Y. et al. Harpagoside inhibits RANKL-induced osteoclastogenesis via Syk-Btk-PLCY2-Ca(2+) signaling pathway and prevents inflammationmediated bone loss. J. Nat. Prod. 78, 2167-2174 (2015).

19. Awsare, N. S., Martin, T. A., Haynes, M. D., Matthews, P. N. \& Jiang, W. G. Claudin11 decreases the invasiveness of bladder cancer cells. Oncol. Rep. 25, 1503-1509 (2011).

20. Agarwal, R. et al. Silencing of claudin-11 is associated with increased invasiveness of gastric cancer cells. PLoS ONE 4, e8002 (2009).

21. Karagiannis, G. S. et al. Collective migration of cancer-associated fibroblasts is enhanced by overexpression of tight junction-associated proteins claudin-11 and occludin. Mol. Oncol. 8, 178-195 (2014).

22. Gao, L. et al. Promoter CpG island hypermethylation in dysplastic nevus and melanoma: CLDN11 as an epigenetic biomarker for malignancy. J. Invest. Dermatol. 134, 2957-2966 (2014).

23. Walesch, S. K., Richter, A. M., Helmbold, P. \& Dammann, R. H. Claudin11 promoter hypermethylation is frequent in malignant melanoma of the skin, but uncommon in nevus cell nevi. Cancers (Basel) 7, 1233-1243 (2015).

24. Patsialou, A. et al. Autocrine CSF1R signaling mediates switching between invasion and proliferation downstream of TGF $\beta$ in claudin-low breast tumor cells. Oncogene 34, 2721-2731 (2015).

25. Mincey, B. A.r Moraghan, T. J. \& Perez, E. A. Prevention and treatment of osteoporosis in women with breast cancer. Mayo Clin. Proc. 75, 821-829 (2000).

26. Fontanges, E., Fontana, A. \& Delmas, P. Osteoporosis and breast cancer. Jt. Bone Spine 71, 102-110 (2004).

27. Khosla, S. Minireview: the OPG/RANKL/RANK system. Endocrinology 142, 5050-5055 (2001)

28. Boyce, B. F. \& Xing, L. Biology of RANK, RANKL, and osteoprotegerin. Arthritis Res. Ther. $\mathbf{9}$ (Suppl 1), S1 (2007).

29. Boyce, B. F. \& Xing, L. Functions of RANKL/RANK/OPG in bone modeling and remodeling. Arch. Biochem. Biophys. 473, 139-146 (2008).

30. Boyce, B. F. \& Xing, L. The RANKL/RANK/OPG pathway. Curr. Osteoporos. Rep. $\mathbf{5}$ 98-104 (2007).

31. Tanaka, M., Kamata, R. \& Sakai, R. Phosphorylation of ephrin-B1 via the interaction with claudin following cell-cell contact formation. EMBO J. 24, 3700-3711 (2005).

32. Dravis, C. \& Henkemeyer, M. Ephrin-B reverse signaling controls septation events at the embryonic midline through separate tyrosine phosphorylationindependent signaling aveneus. Dev. Biol. 355, 138-151 (2011). 
33. Tanaka, M., Kamata, R. \& Sakai, R. EphA2 phosphorylates the cytoplasmic tail of Claudin-4 and mediates paracellular permeability. J. Biol. Chem. 280, 42375-42382 (2005).

34. Bhavaniprasad, V., Prabhu Dass, J. F. \& Jayanthi, S. Activation mechanism of claudin-4 by ephrin type-A receptor 2: a molecular dynamics approach. Mol. Biosyst. 9, 2627-2634 (2013).

35. Shang, X., Lin, X.\& Howell, S. B. Claudin-4 controls the receptor tyrosine kinase EphA2 pro-oncogenic switch through $\beta$-catenin. Cell Commun. Signal. 12, 59 (2014).
36. Zhao, C. et al. Bidirectional ephrinB2-EphB4 signaling controls bone homeostasis. Cell Metab. 4, 111-121 (2006).

37. Davy, A. \& Soriano, P. Ephrin signaling in vivo: look both ways. Dev. Dyn. 232 1-10 (2005).

38. Allan, E. H. et al. EphrinB2 regulation by PTH and PTHrP revealed by molecular profiling in differentiating osteoblasts. J. Bone Miner. Res. 23, 1170-1181 (2008). 